\title{
A humanized mouse model to study asthmatic airway inflammation via the human IL-33/IL-13 axis
}

Ryoji Ito, ${ }^{1}$ Shuichiro Maruoka, ${ }^{2}$ Kaori Soda, ${ }^{2}$ Ikumi Katano, ${ }^{1}$ Kenji Kawai, ${ }^{1}$ Mika Yagoto,, Asami Hanazawa, ${ }^{1}$ Takeshi Takahashi, ${ }^{1}$ Tomoyuki Ogura, ${ }^{1}$ Motohito Goto, ${ }^{1}$ Riichi Takahashi, ${ }^{1}$ Shota Toyoshima, ${ }^{3}$ Yoshimichi Okayama, ${ }^{3}$ Kenji Izuhara, ${ }^{4}$ Yasuhiro Gon, ${ }^{2}$ Shu Hashimoto, ${ }^{2}$ Mamoru Ito, ${ }^{1}$ and Satoshi Nunomura ${ }^{4}$

'Central Institute for Experimental Animals (CIEA), Kanagawa, Japan. ${ }^{2}$ Division of Respiratory Medicine, Nihon University School of Medicine, Tokyo, Japan. ${ }^{3}$ Allergy and Immunology Research Project Team, Research Institute of Medical Science, Center for Institutional Research and Medical Education, Nihon University School of Medicine, Tokyo, Japan. ${ }^{4}$ Division of Medical Biochemistry, Department of Biomolecular Sciences, Saga Medical School, Saga, Japan.

Asthma is one of the most common immunological diseases and is characterized by airway hyperresponsiveness (AHR), mucus overproduction, and airway eosinophilia. Although mouse models have provided insight into the mechanisms by which type-2 cytokines induce asthmatic airway inflammation, differences between the rodent and human immune systems hamper efforts to improve understanding of human allergic diseases. In this study, we aim to establish a preclinical animal model of asthmatic airway inflammation using humanized IL-3/CM-CSF or IL-3/CM-CSF/IL-5 Tg NOD/Shi-scid-IL2ry null (NOG) mice and investigate the roles of human type-2 immune responses in the asthmatic mice. Several important characteristics of asthma - such as AHR, goblet cell hyperplasia, T cell infiltration, IL-13 production, and periostin secretion - were induced in IL-3/CM-CSF Tg mice by intratracheally administered human IL-33. In addition to these characteristics, human eosinophilic inflammation was observed in IL-3/GM-CSF/IL-5 Tg mice. The asthmatic mechanisms of the humanized mice were driven by activation of human Th2 and mast cells by IL-33 stimulation. Furthermore, treatment of the humanized mice with an anti-human IL-13 antibody significantly suppressed these characteristics. Therefore, the humanized mice may enhance our understanding of the pathophysiology of allergic disorders and facilitate the preclinical development of new therapeutics for IL-33-mediated type-2 inflammation in asthma.

Authorship note: RI and SM contributed equally to this work.

Conflict of interest: The authors have declared that no conflict of interest exists.

License: Copyright 2018, American Society for Clinical Investigation.

Submitted: April 10, 2018 Accepted: September 26, 2018 Published: November 2, 2018

\section{Reference information:} JCI Insight. 2018;3(21): e121580. https://doi.org/10.1172/jci. insight.121580.

\section{Introduction}

Asthma, a chronic immune disorder of the bronchus, is characterized by airway hyperresponsiveness (AHR) and remodeling, mucus hyperproduction, and leukocyte inflammation. Despite treatment with inhaled corticosteroids plus a second controller (long-acting $\beta 2$ agonist, leukotriene receptor antagonist, or theophylline), many patients with asthma exhibit sustained, uncontrolled allergic airway inflammation (1-3). Such individuals belong to the high-risk group of asthmatic patients and have a poor quality of life $(4,5)$. Th2 cells, mast cells, basophils, and type-2 innate lymphoid cells (ILC2) produce Th2 cytokines, such as IL-4, IL-5, and IL-13, which contribute to the initiation and exacerbation of asthmatic airway inflammation. The airway epithelial cell-derived Th2-promoting cytokines IL-33, thymic stromal lymphopoietin (TSLP), and IL-25 activate these immune cells following exposure to allergens (6-10).

Therapeutic monoclonal antibodies targeting IL-4 and/or IL-13 can be effective in patients with uncontrolled asthma (11). In a clinical trial, dupilumab, an anti-IL-4 receptor $\alpha$ monoclonal antibody, which inhibits both IL-4 and IL-13 signaling, reduced the asthma symptoms by inhibiting type- 2 inflammation and pulmonary eosinophilia $(12,13)$. Therefore, establishment of an in vivo model that can reconstitute human type-2 immune responses is important for the development of therapeutics effective against type-2 inflammation in asthma patients.

Animal models enable elucidation of the pathological and physiological mechanisms underlying disease and the preclinical testing of new drugs (14). For the induction of asthmatic airway inflammation, 
mice are easily sensitized to various antigens, including ovalbumin (15), house dust mite (HDM) (16, 17), cockroach (18), Aspergillus fumigatus (19), and ragweed extracts (20). After sensitization, inhalation of these allergens induces the expression of type- 2 cytokines in the lungs, resulting in antigen-specific IgE, eosinophilia, and AHR. Although murine models have enabled investigation of the pathogenic role of inflammatory cells, few in vivo models recapitulate the asthmatic responses mediated by human immune cells.

Humanized mice reconstituted with the human immune system are useful for investigating human immunology and establishing models of human immune diseases. Over the past decade, we and other groups have reported several severely immunodeficient mouse strains, such as NOD/Shi-scid-IL2r $\gamma^{\text {null }}$ (NOG) (21-23), NOD/LtSz-scid IL2ry $\gamma^{\text {null }}$ (NSG) (24, 25), and BALB/c Rag2 IL2ry null (BRG) (26), which facilitate engraftment and differentiation of human immune cells after hematopoietic stem cell (HSC) transplantation. However, these strains do not exhibit differentiation of human myeloid lineage cells (including mast cells and eosinophils) and, therefore, are not appropriate models of human allergic diseases.

Recently, we developed a next-generation NOG strain into which the human IL-3 and GM-CSF genes were introduced (NOG IL-3/GM Tg) $(27,28)$. In this model, human myeloid cells — including mast cells, basophils, and eosinophils - differentiate and maturate. Furthermore, a mast cell-mediated passive cutaneous anaphylaxis (PCA) reaction in response to antigen-specific human IgE can be induced.

In this study, we used NOG IL-3/GM Tg and NOG IL-3/GM/IL-5 Tg mice, which is a newly established mouse strain, to induce human eosinophil differentiation from HSC. We established a human type-2 cytokine-induced asthma model by intratracheal administration of human IL-33, and the mice exhibited characteristics similar to those of human asthma. This is the first humanized mouse model to our knowledge that recapitulates the pathology of human asthma, and it will facilitate the development of potentially novel therapeutic agents in preclinical studies.

\section{Results}

Infiltration of human T cells and mast cells into the lungs of the humanized mice. First, we confirmed that the chimeric condition of human immune cells was adequate for this study at 12 weeks after transfer of human CD34+ HSC (data not shown). To induce asthmatic airway inflammation in hu-IL-3/GM Tg mice, recombinant human IL-33 was intratracheally administered for 3 consecutive days, and the bronchoalveolar lavage fluid (BALF) and lungs were analyzed 1 day after the final administration of IL-33 (Figure 1A). H\&E staining showed marked leukocyte infiltration into the bronchus of IL-33-treated hu-IL-3/GM Tg mice. The majority of infiltrated leukocytes were human $\mathrm{CD}^{+} \mathrm{T}$ cells; large numbers of $\mathrm{MCC}^{+}$mast cells were also present (Figure 1, B-D). In the analysis of $\mathrm{T}$ cell subsets, $\mathrm{CD} 4^{+} \mathrm{T}$ cells expanded preferentially in BALF compared with peripheral blood (PB) (Figure 1E). Although the frequency of $\mathrm{CD}^{+} / \mathrm{CD} 8^{+} \mathrm{T}$ cells did not change after IL-33 treatment (Figure $1 \mathrm{~F}$ ), the cell number of $\mathrm{CD} 4^{+}$and $\mathrm{CD} 8^{+} \mathrm{T}$ cell subsets increased after IL-33 treatment in lungs but not in the spleen of hu-IL-3/GM Tg mice (Figure 1G). These data demonstrated that the human $\mathrm{T}$ cells and mast cells were dominantly infiltrated into the airway of hu-IL-3/GM Tg mice with IL-33 treatment, and both human $\mathrm{CD}^{+}$and -CD8 ${ }^{+} \mathrm{T}$ cells in lungs proliferated in response to IL-33.

Production of human cytokines in the humanized mice. We next investigated the levels of human cytokines and chemokines in the lungs of IL-33-treated humanized mice. IL-13 and IL-5 showed high level in the BALF of IL-33-treated hu-IL-3/GM Tg mice but not in the BALF of the other mouse strains (Figure 2A). CCL2 and IL-8 levels were slightly — but not significantly — increased, while other cytokines were not detected. Because IL-13 is a key factor in the development of asthmatic disease, we investigated the cell subsets responsible for IL-13 production in the lungs of the humanized mice. After IL-33 treatment, a large number of $\mathrm{CD}^{+} \mathrm{T}$ cells and a few CD203c $\mathrm{c}^{+}$mast cells were present in the BALF of hu-IL-3/GM Tg mice. After phorbol 12-myristate 13-acetate (PMA) and ionomycin (IM) stimulation, human IL-13 expression was significantly upregulated in BALF $\mathrm{T}$ cells and mast cells compared with that in splenic $\mathrm{T}$ cells and mast cells (Figure 2B). In rodent models, ILC2 plays a crucial role in asthmatic airway inflammation by producing type- 2 cytokines, such as IL-13 and IL-5. Thus, we assayed ILC2 in the lungs of the humanized mice. The scarce population of $\mathrm{CD} 45^{+} \mathrm{Lin}^{-} \mathrm{CD} 161^{+} \mathrm{CRTH} 2^{+}$human ILC2 was detected in the lungs of huIL-3/GM Tg mice regardless of IL-33 stimulation (Figure 2C). However, the cell number of ILC2 slightly increased after IL-33 stimulation (Figure 2D). These results suggest that the human T cells and mast cells are likely responsible for the initiation of asthmatic airway inflammation by producing human IL-13, and human ILC2 also might be responded by IL-33 stimulation. 
A
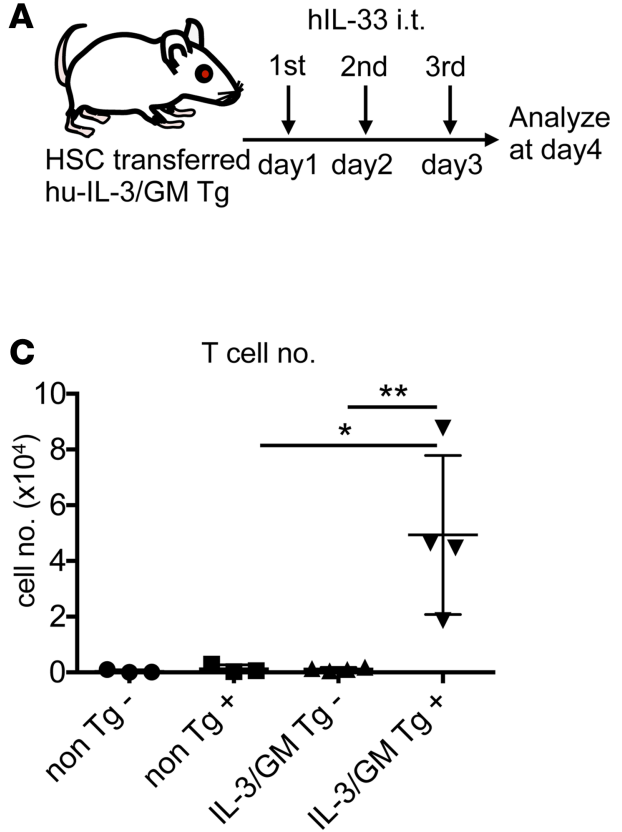

E $\quad \mathrm{CD} 8$ $=\mathrm{CD} 4$

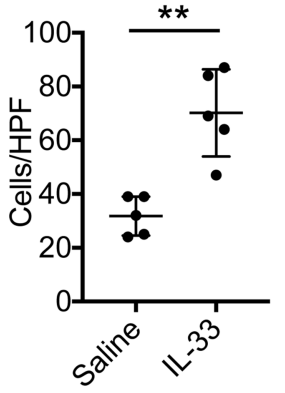

B
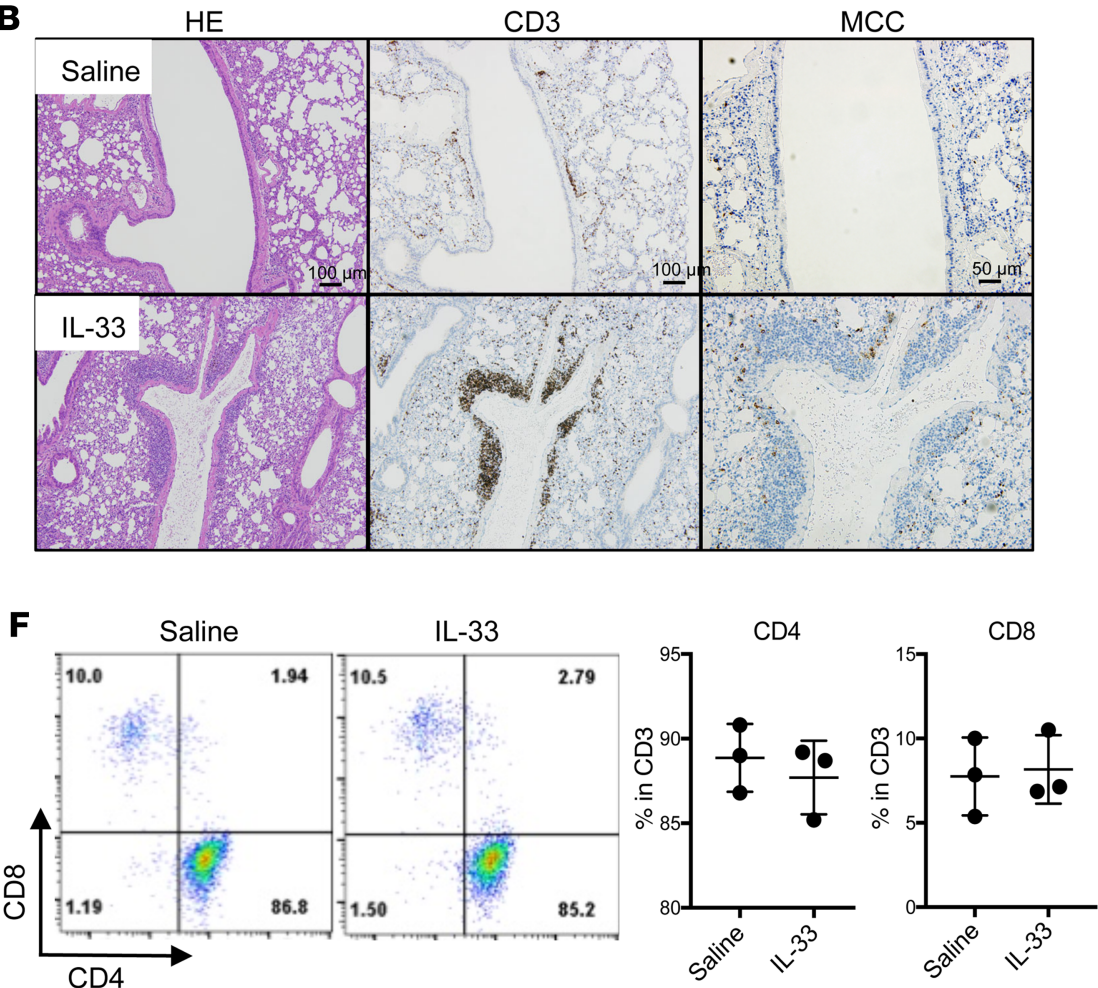

G

Lungs CD4 CD8

Spleen
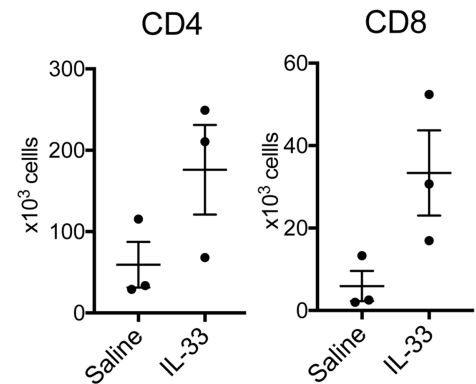

CD8

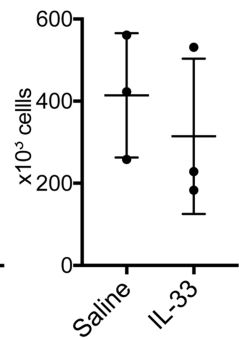

Figure 1. Development of a human asthma model using HSC-transferred NOG IL-3/GM-CSF Tg mice. (A) Schematic of induction of asthmatic airway inflammation using HSC-transferred IL-3/GM Tg mice and intratracheal (i.t.) administration of recombinant human IL-33. (B) Histology of the lungs of hu-IL-3/GM Tg mice after administration of IL-33. Lung sections from hu-IL-3/GM Tg mice treated with or without IL-33 were stained with $\mathrm{H \& E}$, as well as anti-CD3 and anti-human mast cell chymase (MCC) antibodies. Each brown dot represents an individual human CD3- or MCC-expressing T cell or mast cell. Representative images from 3 mice are shown. (C) Number of human T cells in BALF of hu-IL-3/GM Tg or non-Tg mice with or without IL-33 administration. (D) Human MCC mast cells were quantified in the lung lesions of hu-IL-3/GM Tg mice. HPF, high-power field. (E) Frequency of CD4 $4^{+}$or $C D 8^{+}$cells among total CD3 ${ }^{+} T$ cells in the BALF or PB of IL-33-treated hu-IL-3/CM Tg mice $(n=4)$. (F) Left panels showed the flow cytometry data of CD4 ${ }^{+}$and CD8 ${ }^{+}$T cells in CD3 $3^{+}$population with or without IL-33 treatment. Right dot plot graphs show the cumulative data of the frequency of CD4+ and CD8 ${ }^{+} T$ cells in the $C D 3^{+}$population with or without IL-33 treatment. (C) Cell number of CD4+ and CD8 ${ }^{+} \mathrm{T}$ cells in lungs and spleen of hu-IL-3/GM Tg mice with or without IL-33 treatment. Data are represented each 3 mice in $\mathbf{F}$ and $\mathbf{G}$. Original magnification, $\times 10$ for H\&E and CD3, and $\times 20$ for MCC. Scale bar: $100 \mu \mathrm{m}$ for H\&E and CD3; $50 \mu \mathrm{m}$ for MCC. Statistical significance was calculated using Student's $t$ test (D, E, and $\mathbf{G})$ and 1-way ANOVA (C). ${ }^{*} P<0.05$ and ${ }^{* *} P<0.005,{ }^{* * *} P<0.0005$, and ${ }^{* * * *} P<0.00005$.

Characteristics of asthma in IL-33-treated humanized mice. Patients with asthmatic airway inflammation typically exhibit mucus hyperproduction by goblet cells in the lungs. To examine mucus production by goblet cells in the lungs of the humanized mice, we performed periodic acid-Schiff (PAS) staining. After administration of IL-33, hu-IL-3/GM Tg mice exhibited mucus hyperproduction and goblet cell hyperplasia, whereas IL-33-administered hu-non-Tg mice and saline-administered hu-non-Tg and hu-IL-3/ GM Tg mice did not (Figure 3, A and B). Expression of periostin, a matricellular protein, is induced by IL-13, and the protein is deposited in the bronchial epithelium of asthma patients $(29,30)$. It is also a serum biomarker of human Th2-high asthma (31). The murine periostin concentration in BALF was significantly increased only in IL-33-administered hu-IL-3/GM Tg mice (Figure 3C); this finding was 

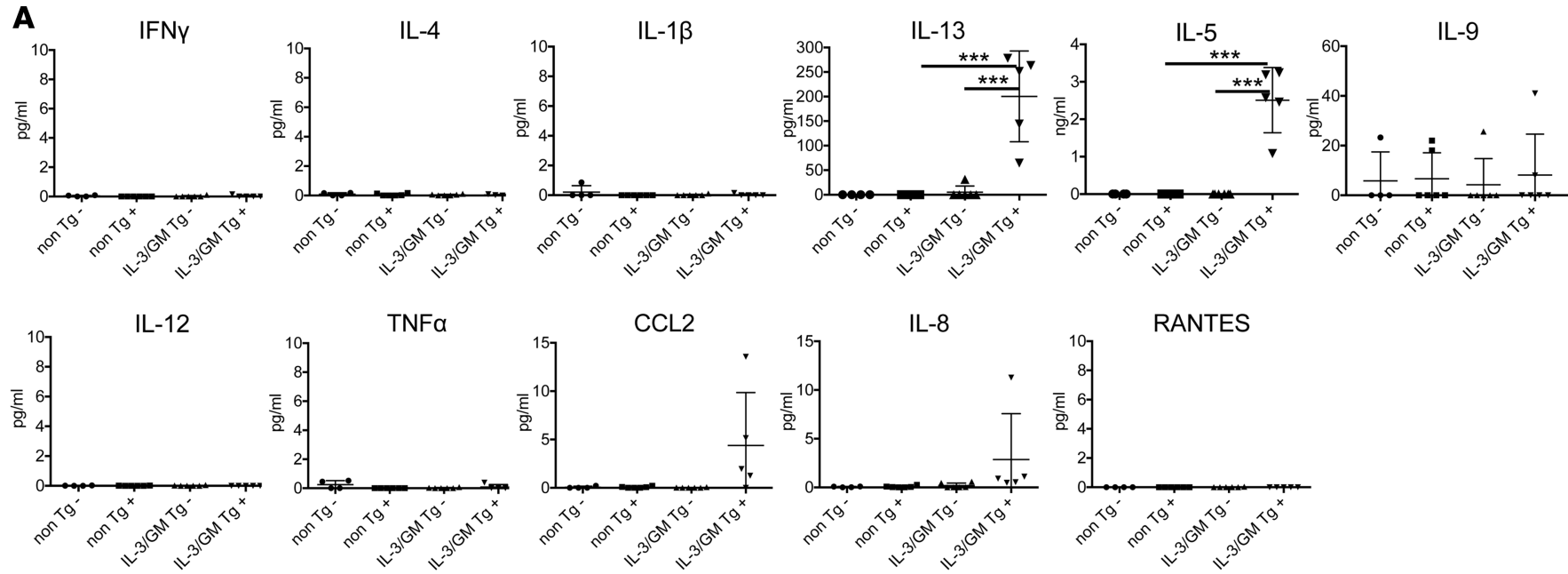

B
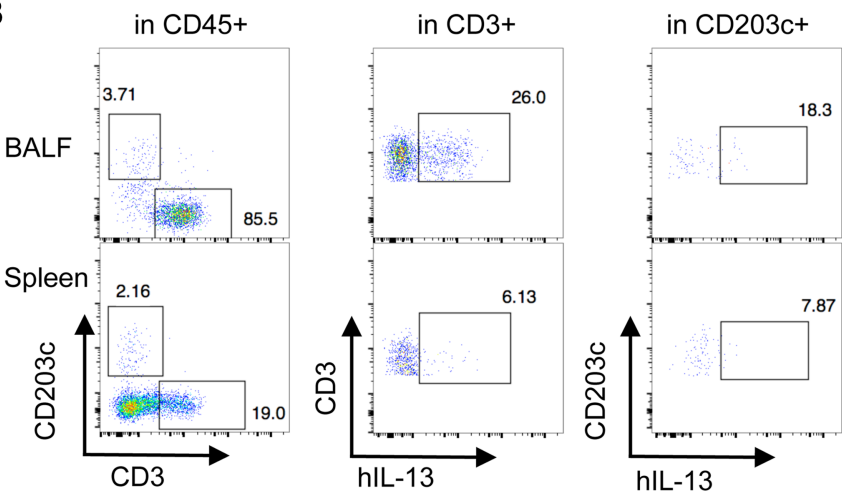

D

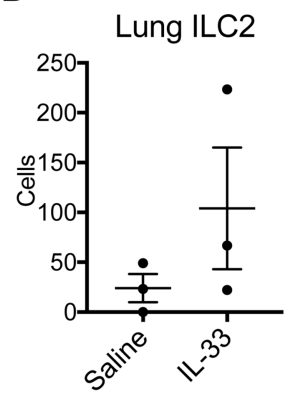

\section{C}

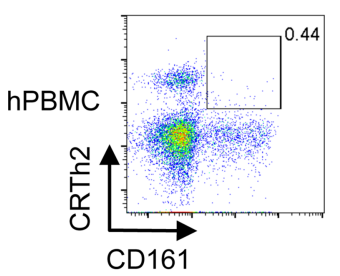

in CD45+Lin-

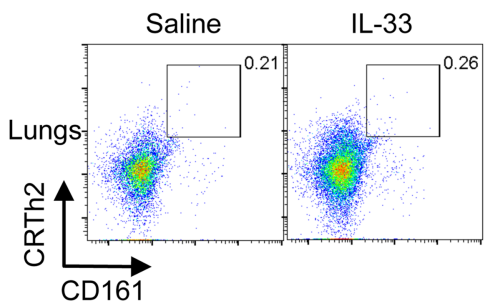

Figure 2. Production of human cytokines and chemokines. (A) Levels of human cytokines and chemokines in the BALF of hu-non-Tg ( $n=4$ or 6$)$ or IL-3/GM Tg mice ( $n=5$ or 6 ) with or without IL-33 treatment were analyzed by cytometric bead array (CBA). (B) Flow cytometry of an intracellular IL-13 cytokine in human $\mathrm{CD}^{+} \mathrm{T}$ cells or CD203C $\mathrm{C}^{+}$mast cells isolated from the BALF and spleen of IL-33-administered hu-IL-3/CM Tg mice. Cells were stained with anti-human CD45, CD3, -CD203c, and -IL-13 antibodies. Representative data from 3 independent experiments are shown. (C) Flow cytometry of human ILC2 in human PBMCs or in the lungs of IL-33-treated hu-IL-3/GM Tg mice $(n=3)$. (D) Cumulative data of cell number of human ILC2 represented in C. Cells were stained with anti-human CD45, -lineage marker, -CD161, and -CRTh2 antibodies. Statistical significance was calculated using 1-way ANOVA (A). ${ }^{* *} P<0.0005$.

confirmed by IHC (Figure 3D). Furthermore, the murine periostin level was highly correlated with that of human IL-13 and human IL-5, reflecting the clinical situation (Figure 3E). However, there was no difference in serum periostin level between hu-IL-3/GM Tg and hu-non-Tg mice, even in the presence of IL-33 stimulation (Supplemental Figure 2; supplemental material available online with this article; https://doi. org/10.1172/jci.insight.121580DS1). We next evaluated AHR in these IL-33-treated hu-IL-3/GM Tg and hu-non-Tg mice by increasing concentration of methacholine inhalation. AHR was significantly higher in IL-33-administered hu-IL-3/GM Tg mice compared with saline-treated $\mathrm{Tg}$ mice when $25-50 \mathrm{mg} / \mathrm{ml}$ methacholine was inhaled (Figure 3F). The level of AHR was no difference in hu-non-Tg mice with or without IL-33 (Figure 3F). Furthermore, highly responsive mice in AHR produced a high level of human IL-5 (Figure 3G). These results indicate that some important characteristics of asthmatic airway inflammation, including AHR, are recapitulated in IL-33-administered hu-IL-3/GM Tg mice. 


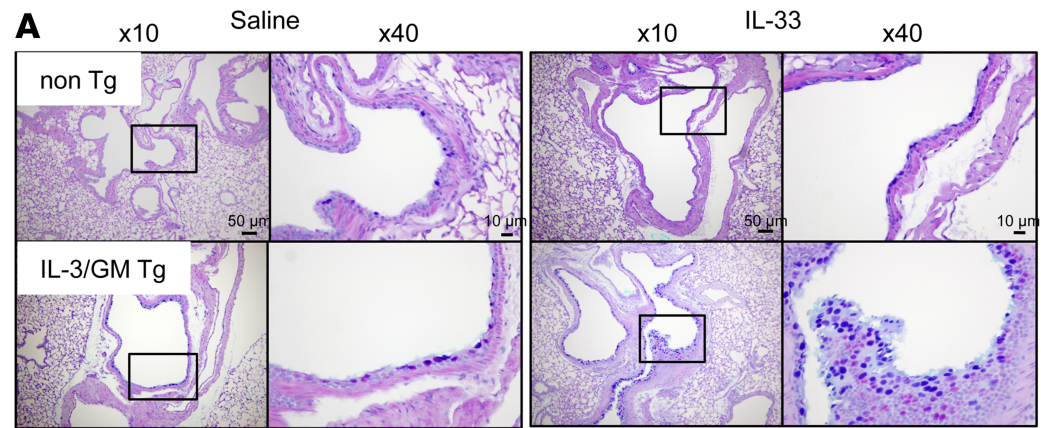

B

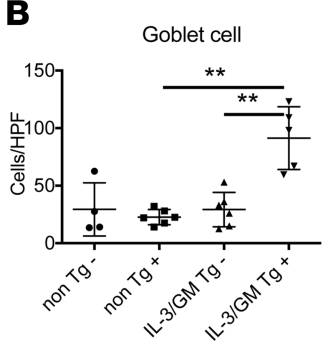

C

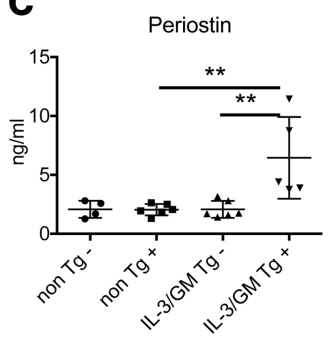

E

D
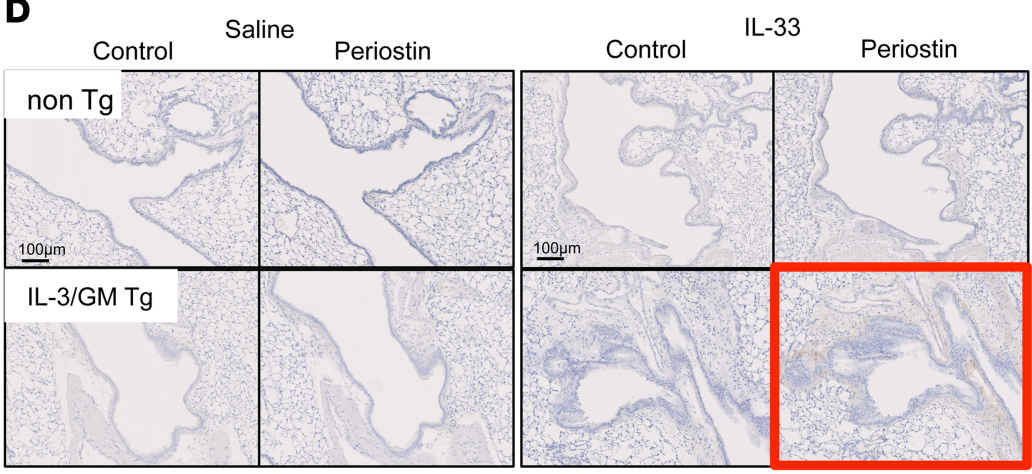

$\mathbf{E}_{300}$
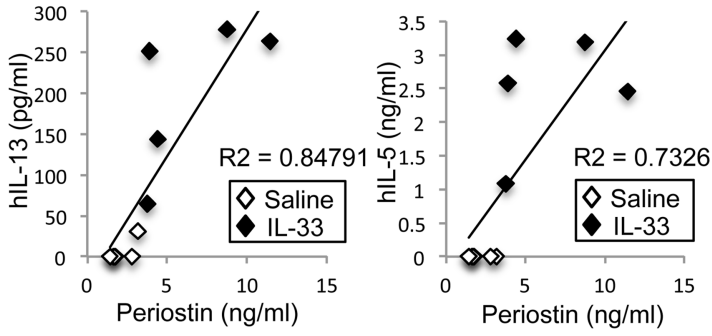

$\mathbf{F}$

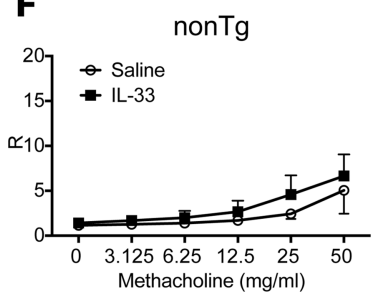

IL-3/GM Tg

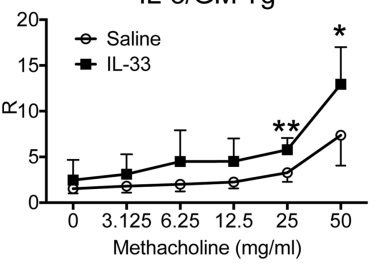

G

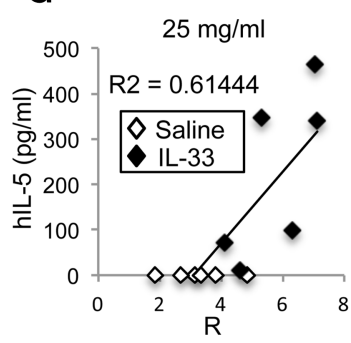

Methacoline inhalation

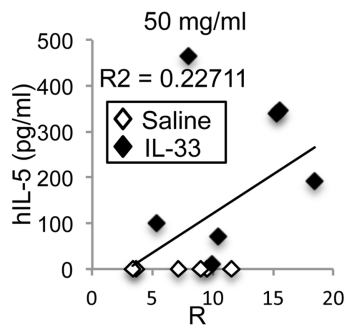

Figure 3. Asthma characteristics in IL-33-treated hu-IL-3/GM Tg mice. (A) Images of murine goblet cells in the lungs of hu-non-Tg and hu-IL-3/GM Tg mice administered saline or IL-33 and stained by Alcian Blue-Periodic acid-Schiff (AB-PAS). (B) Enumeration of PAS-positive goblet cells in the lungs. HPF, high-power field. (C) Murine periostin levels in BALF were measured by ELISA. (D) IHC of periostin. Lung sections from hu-non-Tg and Tg mice with or without IL-33 treatment were stained with anti-mouse periostin or its isotype control. Representative data from each 4 or 5 mice/group are shown. Red-outline represents that periostine was positively stained. (E) Relationship between IL-13 ( $x$ axis) and periostin ( $y$ axis) and IL-5 ( $x$ axis) and periostin ( $y$ axis) levels in the BALF of Tg mice treated with saline $(n=6)$ or IL-33 $(n=5)$. (F) Mice received increasing doses of methacholine as indicated. Airway resistance $(\mathrm{R})$ after human IL-33 or saline treatment was measured using the FinePointe airway resistance analysis system. Three hu-non-Tg mice and 6 hu-IL-3/GM Tg mice were used. (C) Relationship between IL-5 ( $x$ axis) and airway resistance (R) ( $y$ axis) in the BALF of Tg mice treated with IL-33. Data are represented 3-6 mice in each group. Original magnification, $x 10$ or $\times 40$ in (A); $\times 10$ in (D). Scale bar: $50 \mu \mathrm{m}$ or $10 \mu \mathrm{m}$ in (A); $100 \mu \mathrm{m}$ in (D). ${ }^{*} P<0.05$ and ${ }^{* *} P<0.005$ compared with saline-treated groups. Statistical significance was calculated using Student's $t$ test (F) and 1-way ANOVA (B and C). Pearson's correlation coefficient $\left(R^{2}\right)$ was used to assess correlations (E and $\left.\mathbf{G}\right)$.

IL-13 inhibition suppresses characteristics of asthma. To determine whether IL-13 inhibition suppresses characteristics of asthma, we administered an anti-human IL-13 antibody to IL-33-administered hu-IL-3/GM Tg mice. Beginning 1 day before IL-33 treatment, an anti-IL-13 antibody was administered to the mice for 4 consecutive days (Figure 4A). As shown in Figure 4B, the number of human leukocytes, especially human $\mathrm{T}$ cells, in the BALF of IL-33-administered hu-IL-3/GM Tg mice significantly decreased after IL-13 antibody treatment (Figure 4B); the number of human eosinophils, mast cells, and basophils also decreased (Figure 4B). The antibody did not affect all subsets of human leukocytes in PB, suggesting that IL-13 neutralization affects only the airway (Figure 4B). We next investigated the effect of treatment with an anti-IL-13 antibody on other characteristics of asthma. Mucus hyperproduction and goblet cell hyperplasia were markedly inhibited by treatment of IL-33-administered hu-IL-3/GM Tg mice with an anti-IL-13 antibody (Figure 4, C and D). The murine periostin level, but not that of human IL-5, in the BALF of Tg mice was also decreased by the blockade of IL-13 (Figure 4, E and F). Because IL-4 activates mast cells and basophils and induces 
A

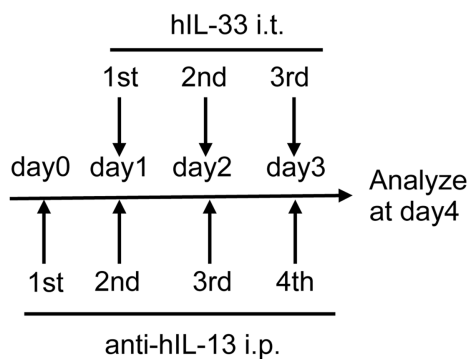

B

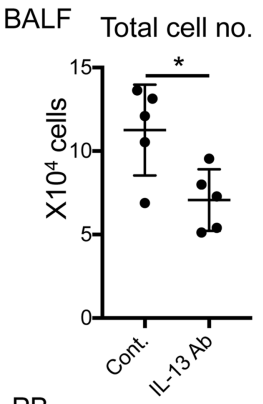

PB

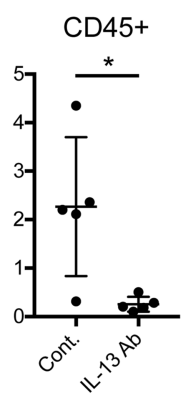

CD45+
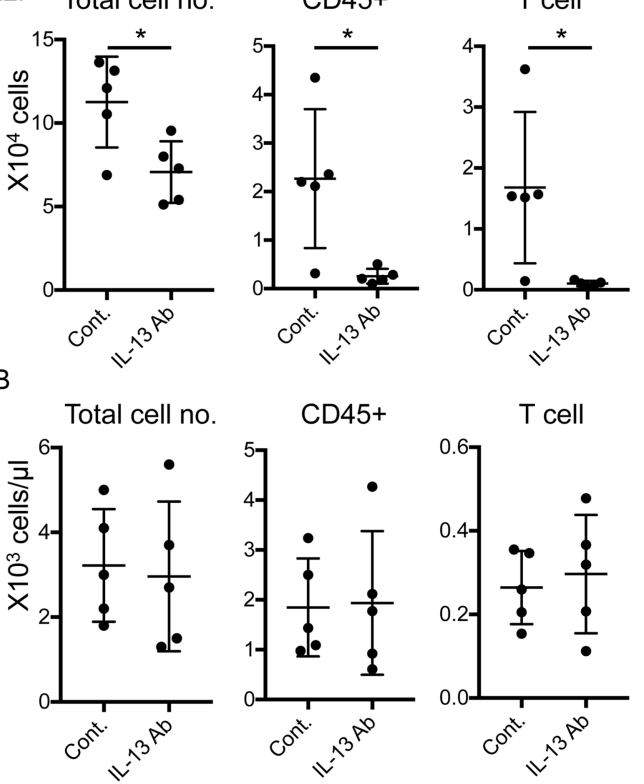

cell

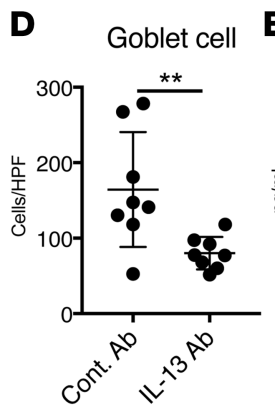

E
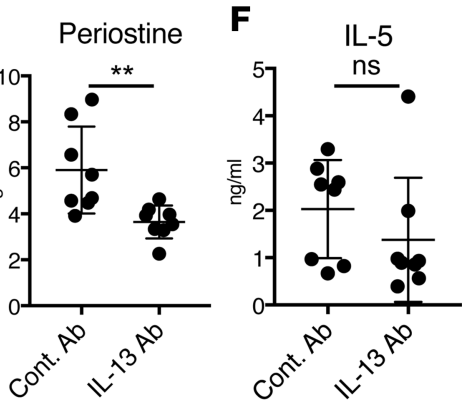

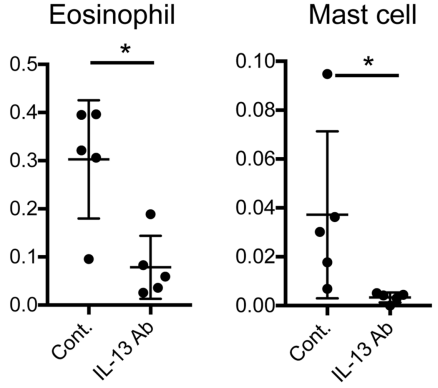

Eosinophil
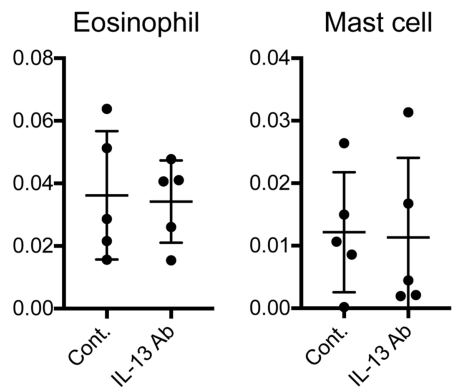

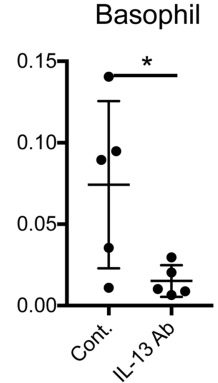

Basophil
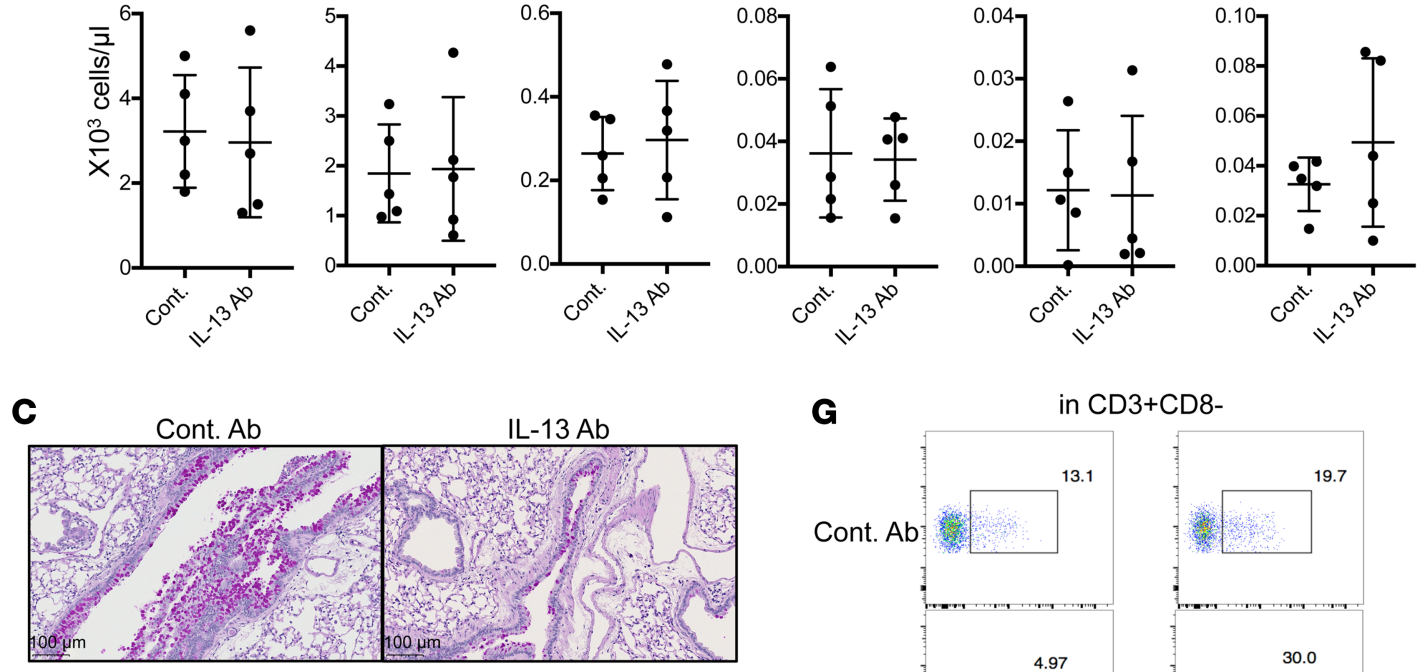

G

in CD3+CD8-

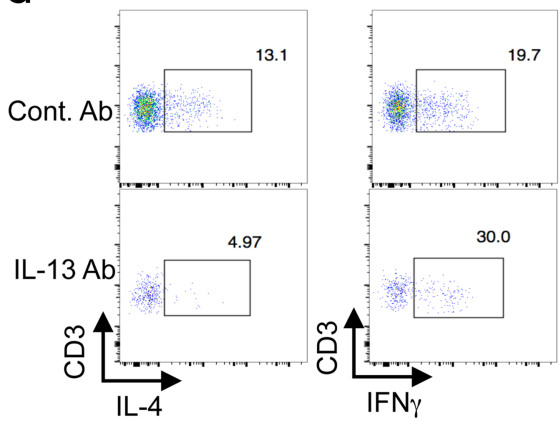

Figure 4. Inhibition of IL-13 suppresses asthmatic airway inflammation. (A) Schematic of the protocol for administration of anti-human IL-13 antibody. (B) Cell numbers of human leukocytes (CD45+ cells), T cells, eosinophils, mast cells, and basophils in the BALF and PB of IL-33-administered hu-IL-3/GM Tg mice treated with anti-human IL-13 $(n=5)$ or rat IgG1 isotype control antibody $(n=5)$. (C) PAS staining of B. Representative data from each 8 mice are shown. (D) Enumeration of goblet cells in C. (E and F) Murine periostin (E) and human IL-5 (F) levels in BALF were measured by ELISA. Each 8 mice/group in these experiments were used in D-F. (C) Intracellular staining of human IL-4 and IFN- $\gamma$. BALF cells were stimulated with phorbol 12-myristate 13-acetate/ionomycin (PMA/IM) for 4 hours and stained with anti-human CD45, $-\mathrm{CD} 3,-\mathrm{CD} 8,-\mathrm{IL}-4$, and $-\mathrm{IFN}-\gamma$ antibodies. CD45+CD3+CD8 ${ }^{-}$cells were defined as CD4 ${ }^{+} \mathrm{T}$ cells due to the decreased level of CD4 expression caused by PMA/IM stimulation. Representative data from 3 independent experiments are shown. Original magnification, $\times 10$. Scale bar: $100 \mu \mathrm{m}$. Statistical significance was calculated using Student's $t$ test $(\mathbf{B}, \mathbf{D}$, and $\mathbf{E}) .{ }^{*} P<0.05$ and ${ }^{* *} P<0.005$.

eosinophil chemotaxis in asthmatic disorders (32), we examined the effect of an anti-IL-13 antibody on Th2 differentiation in the humanized asthma model. Although human CD4+ $\mathrm{T}$ cells expressed IL-4 and IFN- $\gamma$ at similar levels in the absence of the anti-IL-13 antibody, IL-4 expression was markedly suppressed in its presence (Figure $4 \mathrm{G}$ ). In contrast, IFN- $\gamma$ expression by $\mathrm{CD} 4^{+} \mathrm{T}$ cells was significantly enhanced (Figure $4 \mathrm{G}$ ). Human mast cells produced IL-4 in control antibody-treated mice; however, few of these cells were detected 
A
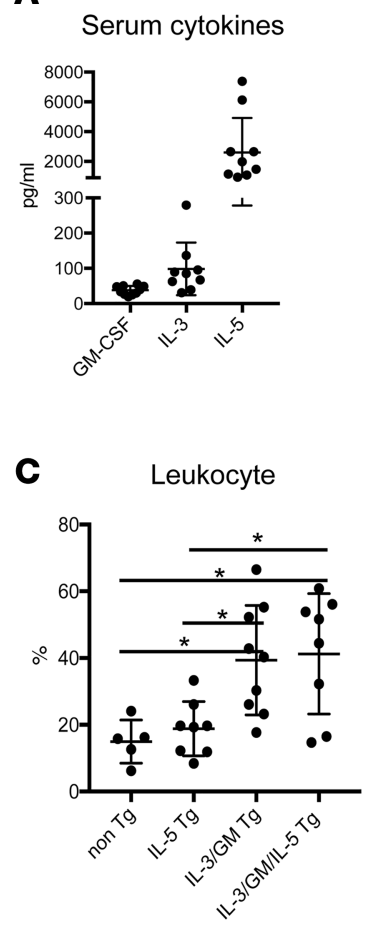

D

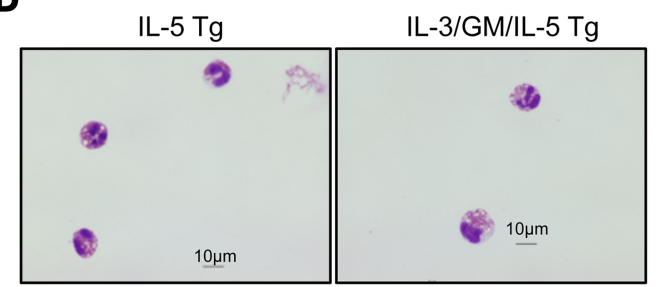

B non Tg IL-5 Tg IL-3/GM TgIL-3/GM/L-5 Tg
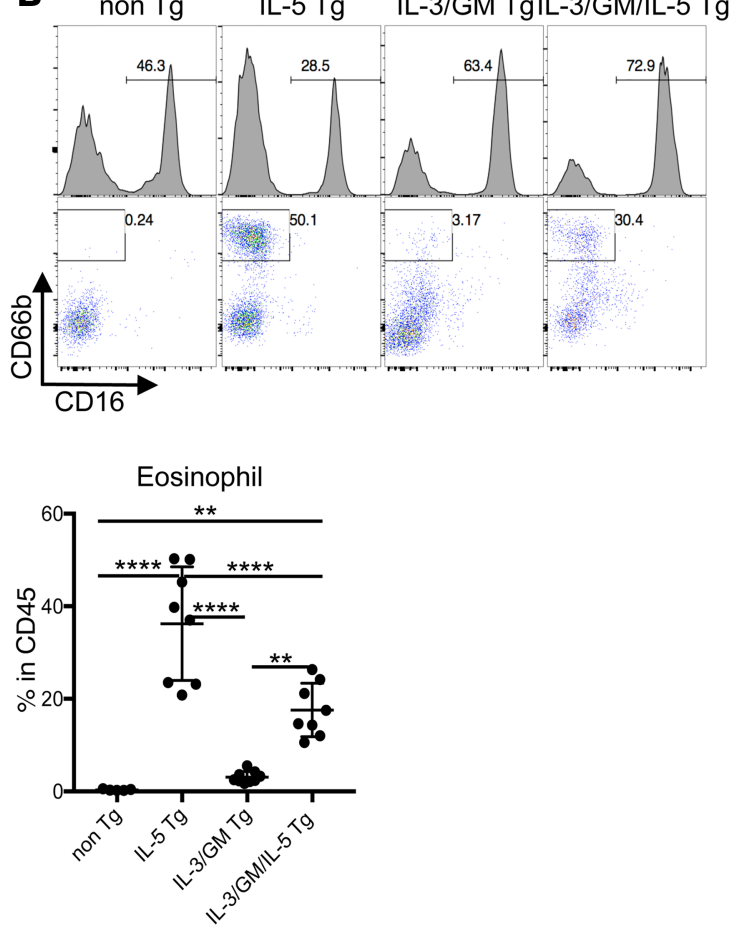

E

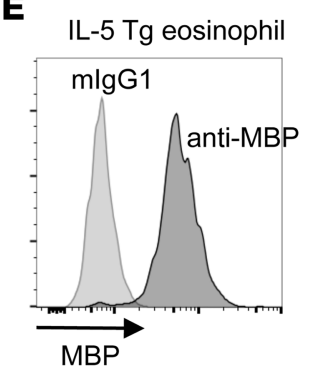

Figure 5. Generation of hu-IL-3/GM-CSF/IL-5 Tg mice. (A) Serum human IL-3, GM-CSF, and IL-5 levels in hu-IL-3/GM-CSF/IL-5 Tg mice $(n=9)$. (B) Flow cytometric analysis of CD45+ leukocytes and CD66b+CD16- eosinophils among CD45 ${ }^{+}$cells in the PB of hu-non-Tg, -IL-5 Tg, -IL-3/GM Tg, and -IL-3/GM/IL-5 Tg mice at 10 weeks after transplantation. (C) Cumulative frequencies of leukocytes and eosinophils. Hu-non-Tg $(n=5),-\operatorname{IL}-5 \operatorname{Tg}(n=8),-\operatorname{IL}-3 / \mathrm{CM} \operatorname{Tg}(n=9)$, and -IL-3/GM/IL-5 Tg $(n=8)$ mice were used. (D) Morphology of human eosinophils. Cytospin samples of CD66b+CD16- eosinophils sorted from the PB of human IL-5 or -IL-3/GM/IL-5 Tg mice were subjected to May-Giemsa staining. (E) Flow cytometry of human Major basic protein (MBP) in CD66b+CD16- eosinophils of hu-IL-5 Tg mice. Mouse IgG1 was used as an isotype control antibody. Representative data from 3 (D) or 4 (E) independent experiments are shown. Original magnification, $\times 40$. Scale bar: $10 \mu \mathrm{m}$. Statistical significance was calculated using 1-way ANOVA (C). ${ }^{*} P<0.05,{ }^{* *} P<0.005$, and ${ }^{* * *} P<0.00005$.

after anti-IL-13 antibody treatment (data not shown). Thus, blockade of IL-13 suppresses Th2 differentiation, resulting in amelioration of the characteristics of asthma, in the humanized asthma model.

Generation of $h u-I L-3 / G M-C S F / I L-5$ Tg mice. Eosinophilic inflammation is a feature of asthma patients with severe symptoms $(33,34)$. In our humanized mice, although human eosinophils were recruited into the lungs of hu-IL-3/GM Tg mice after IL-33 administration (Figure 4B), there is no significant level for the pathogenesis of eosinophilic inflammation. To establish a model of human eosinophilic airway inflammation, we generated NOG IL-3/GM/IL-5-triple Tg mice by introducing the human IL-5 gene into IL-3/ GM Tg mice. The serum human IL-5 level in IL-3/GM/IL-5 Tg mice was markedly higher than that of other cytokines (Figure 5A). After humanization, in addition to significant chimerism of human CD45+ cells based on the IL-3/GM background, a large number of human CD66 $\mathrm{b}^{+} \mathrm{CD} 16^{-}$eosinophils were present in the PB of hu-IL-3/GM/IL-5 Tg mice (Figure 5, B and C). To analyze the morphology of human eosinophils in hu-IL-5 Tgor hu-IL-3/GM/IL-5 Tg mice, we sorted the CD66 $\mathrm{b}^{+} \mathrm{CD} 16^{-}$cell population 
from PBMCs and performed Giemsa staining. Cells that contained red intracellular granules and lobulated nuclei were considered human eosinophils (Figure 5D). Major basic protein (MBP), a cationic protein prestored in eosinophil granules, contributes to allergic responses (35). Human MBP was expressed in the eosinophils of hu-IL-5 Tg mice, which may thus have the functionality of human eosinophils (Figure 5E). These results demonstrate that human eosinophils expressing appropriate surface molecules were highly differentiated in hu-IL-5 and hu-IL-3/GM/IL-5 Tg mice and suggest that these models might be representative of human asthma with eosinophilic inflammation.

Eosinophilic airway inflammation in hu-IL-3/GM/IL-5-triple Tg mice. We intratracheally administered IL-33 to hu-IL-3/GM/IL-5-triple Tg mice to investigate their utility as a model of eosinophilic inflammation. Characteristics of asthma, such as goblet cell hyperplasia, IL-13 production, and AHR, were observed in hu-IL-3/GM/IL-5 triple Tg mice (Figure 6, A-D). Total human IgE antibody in BALF was detected only in a small number of IL-3/GM Tg or IL-3/GM/IL-5 Tg mice, regardless of IL-33 stimulation, suggesting that IL-33 does not affect the IgE production (Supplemental Figure 3). In the BALF of hu-IL-3/GM/ IL-5-triple Tg mice, although total cell number did not increase, human eosinophils, together with murine eosinophils, predominated in the lungs after induction (Figure 6, E and F). In contrast, eosinophils did not infiltrate into the lungs of hu-non-Tg or hu-IL-3/GM Tg mice (Figure 6F). Human and mouse eotaxins are CC-chemokines produced by lung epithelial cells that recruit eosinophils to sites of inflammation in asthmatic disorders (36). The BALF levels of murine eotaxin-1 and -2 (CCL11 and CCL24) were significantly increased in hu-IL-3/GM and hu-IL-3/GM/IL-5 Tg mice after IL-33 administration; in contrast, the levels of both human eotaxins were negligible in all of the mouse strains (Figure 6G). Furthermore, we evaluated human eosinophil-derived neurotoxin $(\mathrm{EDN})$, which is one of the major cationic proteins contained in eosinophil granules, in BALF of the humanized mice. A high level of human EDN was observed in IL-33treated hu-IL-3/GM and hu-IL-3/GM/IL-5 Tg mice (Figure 6H). These results suggest that the human eosinophilic airway inflammation can be recapitulated in hu-IL-3/GM/IL-5-triple Tg mice, and these human eosinophils migrate toward eotaxin-producing airway epithelial cells in an IL-33-dependent manner.

We next intratracheally administered HDM, the antigen is the most common cause of human asthma, to induce airway inflammation in humanized mice. Human IL-5 Tg, IL-3/GM Tg, IL-3/GM/IL-5 Tg, and non-Tg mice were treated with HDM once a week for 3 weeks and analyzed 3 days after the final administration (Figure 7A). We found that the goblet cells tend to increase in lungs by HDM treatment, but it did not show significant differences (Figure 7B). All the human cytokines, including IL-33, were not produced by the treatment in almost all mice (Figure 7, C and D), suggesting human immune cells do not respond to HDM. However, human eosinophils significantly infiltrated into lungs of IL-3/GM/IL-5 Tg mice after HDM treatment (Figure 7E). Similar to the IL-33 treatment, murine eotaxin-1 and -2 were likely produced in HDM-treated IL-3/GM and IL-3/GM/IL-5 Tg mice, but they have no significant differences (Figure 7F). Therefore, the HDM-induced airway inflammation model showed weak characteristics of asthma compared with the IL-33-induced model using hu-IL-3/GM/IL-5-triple Tg mice, but human eosinophilic inflammation may be recapitulated through the murine eotaxin-dependent mechanisms.

\section{Discussion}

In the present study, we generated a potentially novel asthma model in which intratracheal administration of human L-33 into humanized NOG IL-3/GM or IL-3/GM/IL-5 Tg mice resulted in asthmatic airway inflammation mediated by human IL-13 produced by human T cells and mast cells. The mice also exhibited mucus hyperproduction from goblet cells, periostin secretion, AHR, and eosinophilic inflammation. Because anti-human IL-13 treatment suppresses these characteristics, humanized models of asthma may be used in preclinical studies for other candidates.

In a murine model of asthma, IL-33 plays a critical role in the pathogenesis of airway inflammation by inducing the production of type-2 cytokines from the IL-33 receptor-expressing (ST2-expressing) cells, such as basophils, mast cells, and ILC2 (37-40). Christianson et al. reported that ILC2 is involved in persistent asthmatic inflammation via a positive feedback circuit with lung epithelial cells (40). IL-33 produced by damaged epithelial cells induces IL-13 secretion by ILC2; this enhances ST2 expression and IL-33 production by epithelial cells. Moreover, Th2 cells have redundancy to this positive feedback mechanism instead of ILC2 (40). Although the mechanisms similar in the cytokine network to the murine models have been observed in our humanized mice, only a small number of human ILC2 was detected even after stimulation with IL-33 (Figure 2, C and D). It is difficult to determine whether those human ILC2 contribute to the pathology of 
A
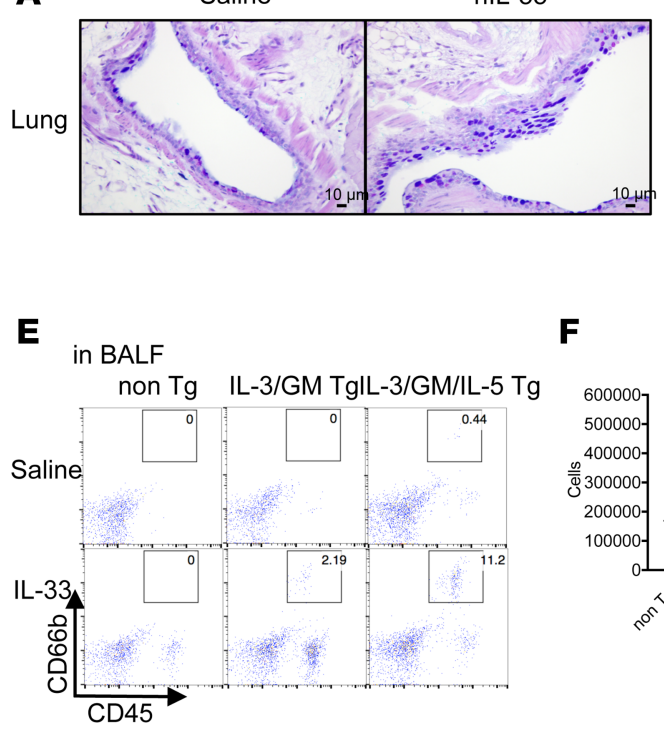

$\mathbf{F}$

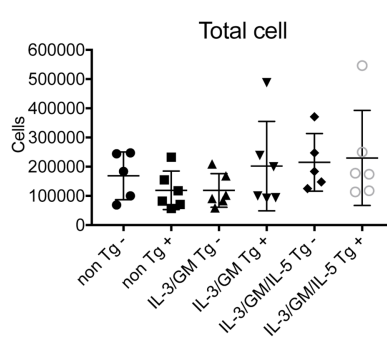

C

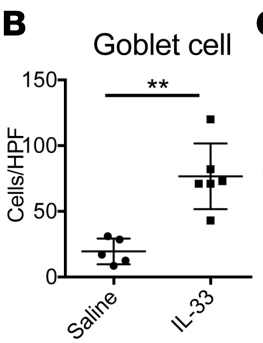

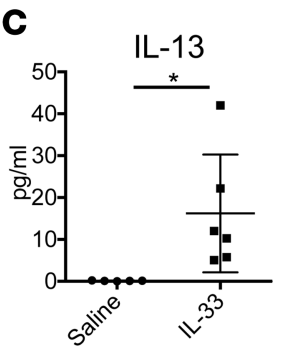

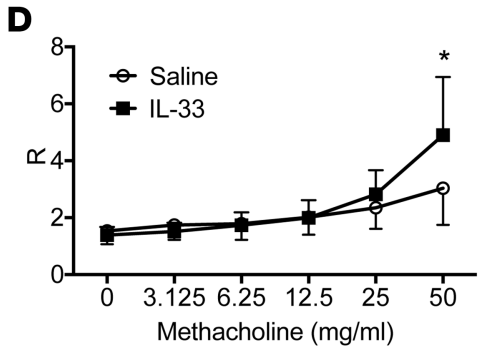

G

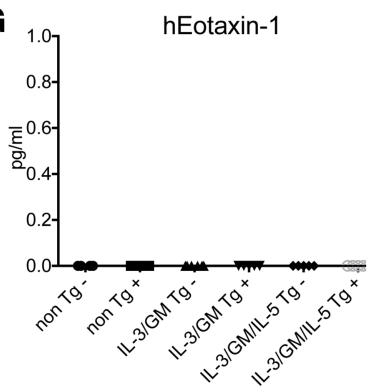

H

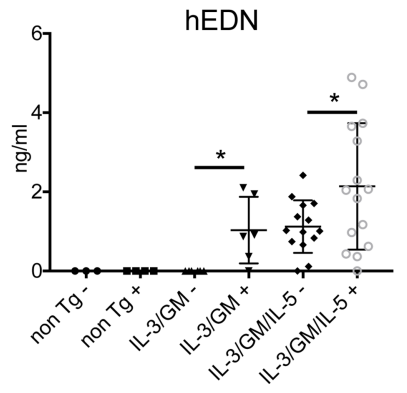

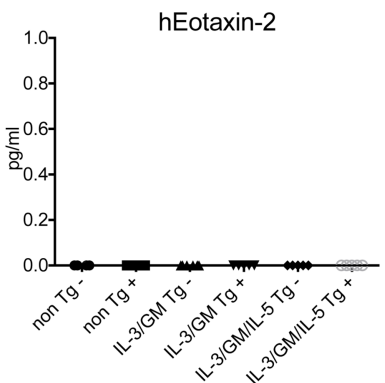
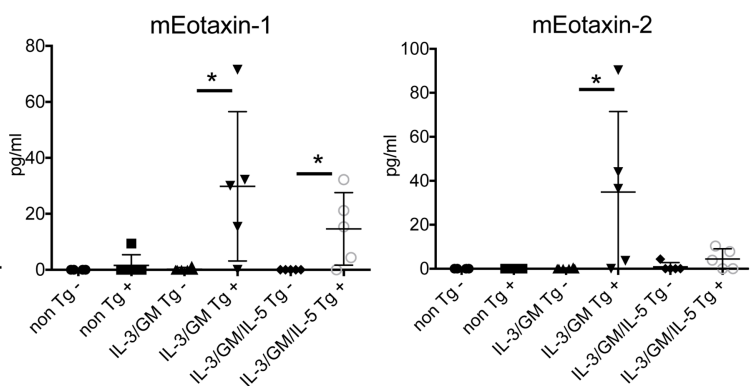

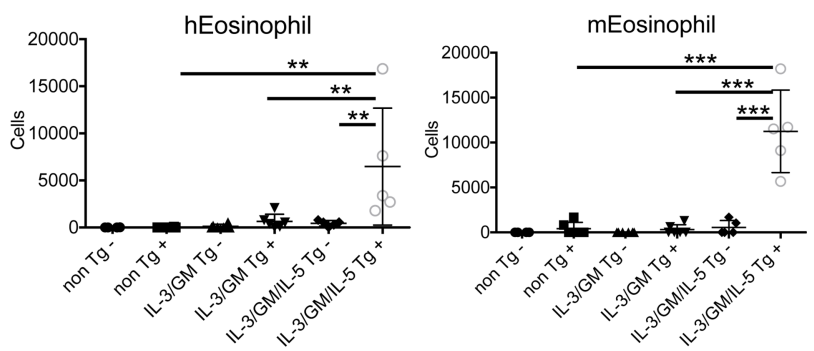

Figure 6. Eosinophilic inflammation in hu-IL-3/GM-CSF/IL-5 Tg mice. (A) Periodic acid-Schiff (PAS) staining of the lungs of hu-IL-3/GM/IL-5 Tg mice treated with or without IL-33. (B) Quantification of goblet cells in bronchus lesions. (C) IL-13 level in the BALF of hu-IL-3/GM/IL-5 Tg mice treated with or without IL-33. (D) Mice received increasing doses of methacholine as indicated. Airway resistance (R) after human IL-33 or saline treatment was measured using the FinePointe airway resistance analysis system. Data are represented each 9 mice/group. (E) Flow cytometry of CD45+CD66b+ eosinophils in the BALF of hu-non-Tg, -IL-3/GM Tg, and -IL-3/GM/IL-5 Tg mice treated with or without IL-33. (F) Quantification of total cells and human and murine eosinophils in BALF. (G and $\mathbf{H}$ ) Human and murine eotaxin-1 and -2 (G) and human eosinophil-derived neurotoxin (EDN) levels (H) in the BALF of hu-non-Tg, -IL-3/CM Tg, and - IL-3/GM/IL-5 Tg mice treated with or without IL-33. Data are represented each 5 or 6 mice in $\mathbf{F}$ and $\mathbf{G} ; 3$ or 4 hu-non-Tg, $6 \mathrm{hu}-\mathrm{IL}-3 / \mathrm{GM}$ Tg, and 14 or 15 hu-IL-3/GM/IL-5 Tg mice were used in $\mathbf{H}$. Original magnification, $\times 40$. Scale bar: $10 \mu \mathrm{m}$. Statistical significance was calculated using Student's $t$ test (B-D) and 1-way ANOVA (F-H). ${ }^{*} P<0.05,{ }^{* *} P<0.005$, and ${ }^{* *} P<0.0005$.

asthma, but there remains some possibility that the inflammation enhanced by ILC2 responded to IL-33 in IL-3/GM-CSF or IL-3/GM-CSF/IL-5 Tg mice. Two studies succeeded in engrafting human ILC2 in immunodeficient mice. Rigas et al. developed a humanized mouse model using NSG mice with adoptive transfer of isolated and cultured human ILC2 (41). Similar to our work, they intratracheally administered human IL-33 into ILC2-reconstituted mice, which resulted in AHR and murine eosinophilic infiltration. However, the inflammatory responses mediated by human cells were limited because mast cells, basophils, and other myeloid lineage cells were not reconstituted. Lim et al. reported that the $\mathrm{Lin}^{-} \mathrm{CD} 7^{+} \mathrm{CD} 127^{+} \mathrm{CD} 117^{+}$population in the cord and adult blood, the fetal liver, and several adult tissues are the progenitor population of all human ILC subsets, including ILC2 (42). These progenitors can be engrafted in humanized mice, where they differentiate into mature ILCs. Therefore, complete reconstitution of human immune cells with a large number of human ILC2 may require transplantation of both $\mathrm{CD} 34^{+}$and ILC progenitors.

IL-13 induces periostin secretion by airway epithelial cells, which contributes to the severity of airway remodeling $(29,43)$. Lebrikizumab is an anti-IL-13 antibody used for severe uncontrolled asthma in patients who failed treatment with an inhaled corticosteroid plus a second controller (44). Lebrikizumab suppresses the symptoms of asthma and improves lung function, especially in patients with high serum periostin levels. 
A

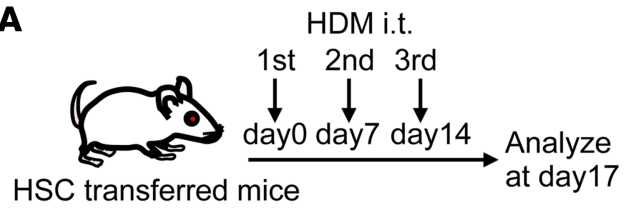

B

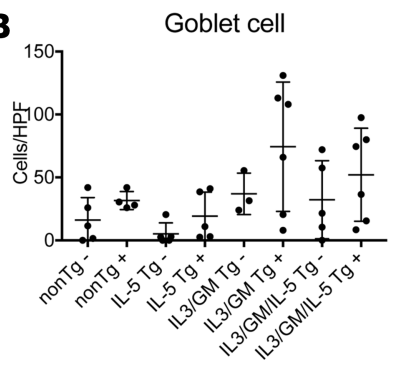

D

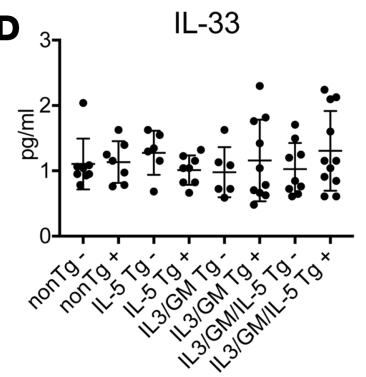

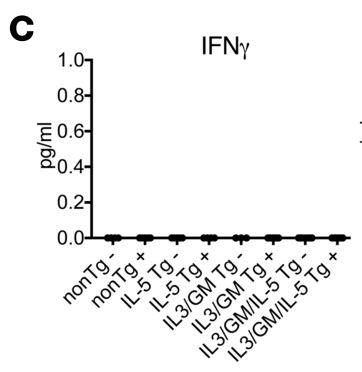

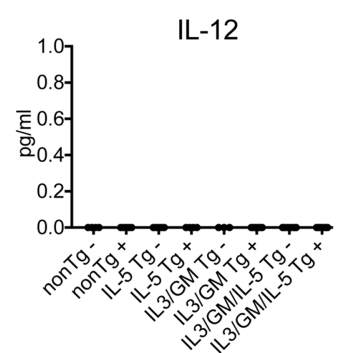

IL-4
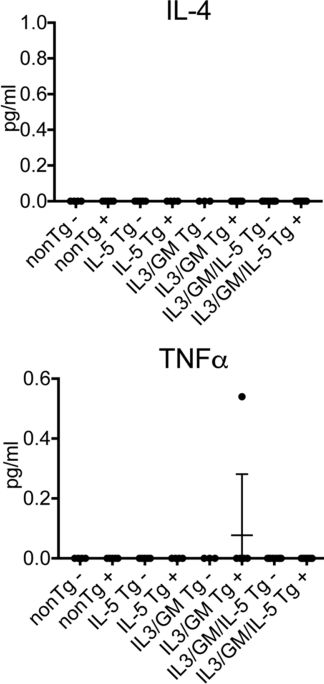
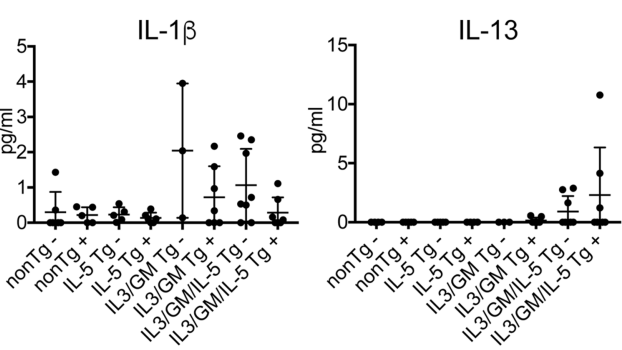

IL-8
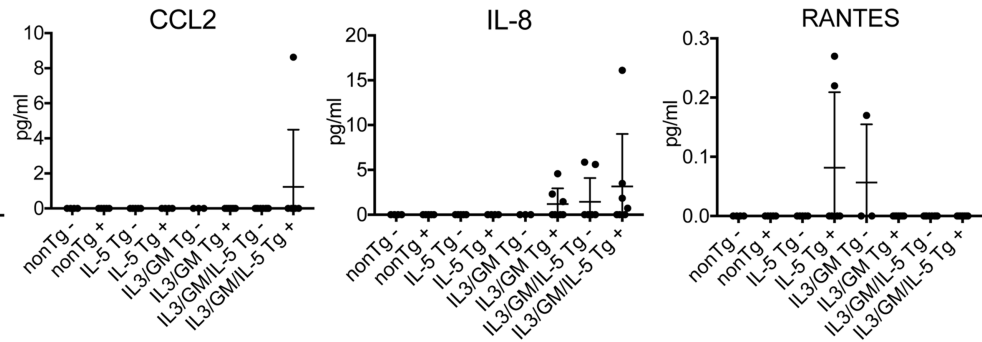

IL-5

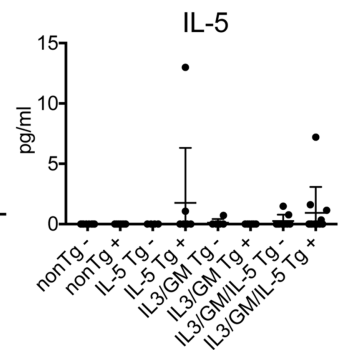

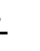

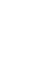
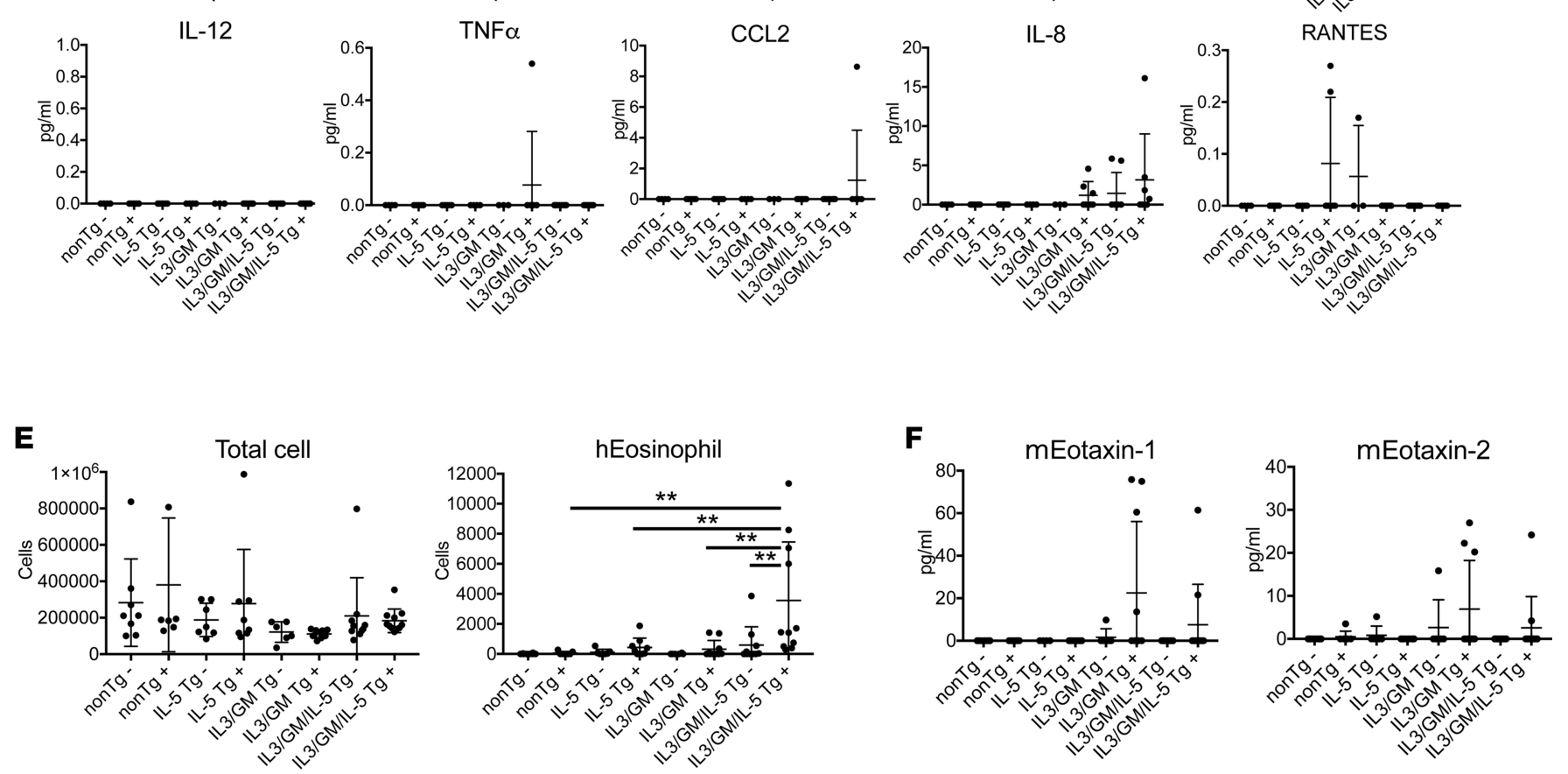

Figure 7. House dust mite-induced (HDM-induced) airway inflammation model. (A) HSC-transferred human IL-5 Tg, -IL-3/GM Tg, -IL-3/GM/IL-5 Tg, and non-Tg mice were i.t. injected HDM at day 0, 7, and 14 and analyzed at day 17. (B) Quantification of goblet cells in lungs of hu-IL-5 Tg( $n=5$ each), hu-IL-3/GM $\operatorname{Tg}(n=3$ or 6$)$, hu-IL-3/GM/IL-5 Tg ( $n=5$ or 6$)$, and hu-non-Tg $(n=5$ or 4$)$ mice with or without HDM treatment. (C) Levels of human cytokines and chemokines in the BALF of hu-IL-5 $\operatorname{Tg}(n=5$ or 6$)$, hu-IL-3/GM Tg ( $n=3$ or 7 ), hu-IL-3/GM/IL-5 Tg ( $n=8$ or 7), and hu-non-Tg ( $n=6$ or 5$)$ mice with or without HDM treatment were analyzed by CBA or ELISA. (D) Levels of human IL-33 in the BALF were analyzed by ELISA. (E) Quantification of total cells and human eosinophils in BALF. (F) Murine eotaxin-1 and -2 in BALF were analyzed by ELISA. Data are represented 6 or 8 human IL-5 Tg, 6 or 10 hu-IL-3/GM Tg, 9 or 11 hu-IL-3/ $\mathrm{GM} / \mathrm{IL}-5 \mathrm{Tg}$, and 8 or 7 hu-non-Tg mice with or without HDM treatment $(\mathbf{E}-\mathbf{F})$. Statistical significance was calculated using 1 -way ANOVA $(\mathbf{E})$. $^{* *} P<0.005$.

In our asthma model, the human IL-13 level was correlated with that of murine periostin, and inhibition of IL-13 suppressed asthmatic airway inflammation, including periostin secretion. Therefore, our data suggest that the mechanisms underlying exacerbations of asthmatic pathology mediated by IL-13 and periostin can be mimicked in humanized mice, which thus have potential for evaluating the efficacy of novel antiasthma drugs.

IL-5 is critical for the growth, activation, and survival of eosinophils. Eosinophils can induce airway remodeling in murine models, as well as in human patients, and IL-5 is a target for therapeutics (45-47). Despite the characteristics of asthma in hu-IL-3/GM Tg mice, little accumulation of human eosinophils in the lungs occurred. We thus generated NOG IL-3/GM-CSF/IL-5 Tg mice by crossing IL-3/GM Tg with IL-5 Tg mice. Strikingly, a considerable number of human eosinophils were present in the BALF of 
these mice after IL-33 administration. This recruitment of human eosinophils might be mediated by murine eotaxins, due to the crossreaction between murine and human. IL-33 causes human T cells and mast cells to produce IL-13, which induces eotaxin production by airway epithelial cells more effectively than IL-4 (48). Because only a small fraction of the eosinophils of hu-IL-3/GM Tg mice are human, human IL-5 is necessary for the expansion of human eosinophils in blood and the induction of eosinophilic inflammation in our humanized mouse model. In one of the major concerns in this model, murine eosinophils were also significantly detected, as well as human eosinophils in BALF of IL-3/GM-CSF/IL-5 Tg mice in the stimulation of human IL-33. Considering the utilization of humanized mice for preclinical therapeutic application, the responses mediated by murine cells are required to be excluded as far as possible. Because murine eosinophils do not function in response to IL-5 in murine IL-5 receptor-KO mice (49), we consider that mice are suitable to overcome this issue and need to combine this model with IL-3/GM-CSF/IL-5 Tg mice in the future.

HDM challenge is most commonly used in murine models of allergic asthma and produces innate proallergic cytokines, TSLP, IL-25, and IL-33 (50). However, mild characteristics of asthmatic airway inflammation have been shown in the humanized mice model after HDM challenge compared with induction by IL-33. We speculated that multiple intratracheal injections of HDM do not fully activate human T cells, due to the defect of lymphopoiesis, especially $\mathrm{T}$ cell differentiation, in humanized mice (51). NOG HLA-DR4 Tg mice (52) combined with IL-3/GM or IL-3/GM/IL-5 Tg mice transferred with HLAmatched $\mathrm{CD}_{3}{ }^{+}$cells may become an improved humanized model mice with differentiation of functional human T cells for HDM or OVA-induced classical allergic asthma.

Our humanized mouse model is limited by intrinsic defects in the class switch machinery of human B cells (53). This hampers the generation of a model of asthma mediated by allergen-specific IgE. An alternative approach is passive induction of asthma using monoclonal IgE against asthma-related antigens, such as HDM. Because we previously reported induction of a PCA reaction mediated by human mast cells, allergen-specific human IgE might trigger more severe reactions in hu-IL-3/GM Tg or IL-3/GM/IL-5 Tg mice; this warrants further investigation.

In conclusion, we developed a potentially novel humanized mouse model of human asthma using hu-IL-3/GM Tg mice treated with IL-33. Various symptoms of asthma - such as goblet cell hyperplasia, IL-13 production, periostin secretion, and AHR - were observed in the mice and were suppressed by administration of an anti-IL-13 antibody. Furthermore, human eosinophilic inflammation was induced in the hu-IL-3/GM/IL-5 Tg mice in a murine eotaxin-dependent manner. The next-generation humanized mouse model reported herein will improve our understanding of the pathogenesis of human asthma and enable evaluation of novel therapeutic agents.

\section{Methods}

Mice. NOG (formal name, NOD.Cg-prkdc scid $i l 2 \mathrm{rg}^{\text {tm } 1 \text { Sug }} / \mathrm{Jic}$ ) (21) and NOD/Shi-scid-IL2r $\gamma^{\text {null }}$ hu-IL-3/ GM-CSF Tg (NOG hIL-3/GM-CSF Tg; formal name, NOD.Cg-prkdcsidil2rg ${ }^{\text {tm } 1 \text { Isug }} \mathrm{Tg}$ [SR $\alpha$-IL-3, CSF2]7-2/Jic/JicTac) (27) mice were previously established in the CIEA. NOG hu-IL-5 Tg(formal name, NOD.Cg-prkd scidil2rg ${ }^{\text {tm } I S u g}$ Tg [CMV-IL-5]1-1/Jic) mice were generated in this study. Human $I L-5$ cDNA was synthesized from mRNA extracted from hu-CD4 ${ }^{+} \mathrm{T}$ cells stimulated with $10 \mathrm{ng} / \mathrm{ml} \mathrm{PMA}$ (MilliporeSigma) and $1 \mu \mathrm{g} / \mathrm{ml}$ IM (MilliporeSigma) in RPMI1640 medium (Thermo Fisher Scientific) for 4 hours at $37^{\circ} \mathrm{C}$ in $5 \% \mathrm{CO}_{2}$. The cDNA was inserted into the $\mathrm{pCMV} \beta$ vector (Takara Bio USA Inc.) and linearized by digestion with EcoRI and HindIII restriction enzymes. Linearized fragments were injected into NOD/NOG F1 mouse embryos. Offspring with the transgenes were backcross-mated to NOG mice to introduce the scid and $I L-2 r \gamma^{\text {null }}$ genes. Then, IL-5 Tg mice were crossed with IL-3/ GM-CSF Tg mice to generate IL-3/GM-CSF/IL-5-triple Tg mice. The mice had access to sterilized food and water ad libitum and were used for human cell transplantation studies at 7-10 weeks of age.

Generation of humanized mice. Commercially available human cord blood-derived CD $34^{+}$cells (AllCells LLC or Lonza) were used in this study. The cells were prepared according to the manufacturer's instructions. Briefly, a cryopreserved $\mathrm{CD} 34^{+}$cell vial was thawed in a water bath at $37^{\circ} \mathrm{C}$ and immediately transferred to RPMI1640 medium (Thermo Fisher Scientific) containing 10\% FCS. After washing with PBS, the viability of CD $34^{+}$HSCs was examined by $2.5 \%$ trypan blue exclusion assay; cells with $>80 \%$ viability were used for transplantation. Human HSCs $\left(4 \times 10^{4}\right.$ to $\left.5 \times 10^{4}\right)$ were i.v. injected into NOG, NOG IL-3/GM Tg, or NOG IL-3/GM/IL-5 Tg mice 1 day after 1.5-2 Gy irradiation using an X-ray system (MBR-1505R; Hitachi Medical). 
Induction of asthmatic airway inflammation. At 14-18 weeks after humanization of NOG, NOG IL-3/GM $\mathrm{Tg}$, and NOG IL-3/GM/IL-5 Tg mice, $1.25 \mu \mathrm{g}$ of recombinant human IL-33 (ATGen Co. Ltd.) or PBS, as a negative control, was intratracheally administered for 3 consecutive days under anesthesia by inhalation of isoflurane (3\%-5\%). One day after the third IL-33 administration, BALF was harvested by injecting $1 \mathrm{ml}$ of PBS into the lungs using a syringe.

For HDM-induced airway inflammation model, HDM (Dermatophagoides pteronyssinus at $100 \mu \mathrm{g} /$ mouse/treatment; GREER Laboratories) was intratracheally injected at days 0 , 7, and 14 under anesthesia, and BALF was harvested at day 17 , as previously described $(50,54)$. BALF samples were analyzed by flow cytometry, ELISA, or cytometric bead array (CBA) as described below.

Flow cytometry. Engraftment and differentiation of human cells in the spleen, PB, and BALF were analyzed by flow cytometry and staining with anti-human antibodies. Cells were prepared using BD PharmLyse (BD Biosciences) to remove RBCs and were incubated in the dark for 20 minutes at $4^{\circ} \mathrm{C}$ in the presence of a mixture of appropriate fluorescently labeled monoclonal antibodies. After washing with PBS, the cells were suspended in propidium iodide (PI) solution (BD Biosciences), subjected to multicolor flow cytometry with FACS Canto (BD Biosciences), and analyzed using FACS Diva software (BD Biosciences). The engraftment ratio of human cells was expressed as the percentage of hu-CD45 cells of total mononuclear cells (mouse plus human), excluding erythrocytes. The following antibodies against cell-surface molecules were used: anti-human CD66b FITC (clone G10F5), -CD8-FITC (clone HIT8a), -CD45-allophycocyanin-Cy7 (APC-Cy7) (clone 2D1), -CD4-phycoerythrin (PE) (clone RPA-T4), -CD203c-PE (clone NP4D6), -c-kit-PE-Cy7 (clone 104D2), -CD14-PE-Cy7 (clone HCD14), -CD3-APC (clone HIT3a), and -CD16-APC (clone 3G8) (all from BioLegend).

For staining of intracellular cytokines, cells isolated from BALF or spleen were stimulated with $10 \mathrm{ng} / \mathrm{ml}$ PMA (MilliporeSigma) and $1 \mu \mathrm{g} / \mathrm{ml} \mathrm{IM}$ (MilliporeSigma) in the presence of $1 \mu \mathrm{g} / \mathrm{ml}$ Brefeldin A (BioLegend) in RPMI1640 medium (Thermo Fisher Scientific) for 4 hours at $37^{\circ} \mathrm{C}$ in $5 \% \mathrm{CO}_{2}$. The cells were fixed and permeabilized in paraformaldehyde fixation buffer (PFA) (BioLegend) and permeabilization wash buffer (BioLegend) before staining. Next, IL-13, IL-4, and IFN- $\gamma$ were stained using anti-human IL-13-APC (clone JES105A2), anti-IL-4 Alexa Fluor 488 (clone 8D4-8), and anti-IFN- $\gamma-$ PE-Cy7 (clone B27) antibodies (BioLegend). Total leukocytes were enumerated using an XT-2000iV automated hematology analyzer (Sysmex Corporation).

For ILC2 staining, lung tissues were minced using scissors and incubated with $1 \mathrm{mg} / \mathrm{ml}$ collagenase IV (Thermo Fisher Scientific) and $2.5 \mathrm{mg} / \mathrm{ml}$ DNase I (MilliporeSigma) in RPMI1640 at $37^{\circ} \mathrm{C}$ for 45 minutes on a shaker (200 rpm). After washing with PBS, mononuclear cells were separated from the interface between $40 \%$ and $65 \%$ Percoll (GE Healthcare) gradient. The cells were immunostained with anti-human lineage cocktail 1 (CD3 [clone SK7], CD14 [clone M 9 P9], CD16 [clone 3G8], CD19 [clone SJ25C1], CD20 [clone L27], and CD56 [clone NCAM16.2]) FITC (BD Biosciences), -CD161-PE (clone NKR-P1A, BioLegend), -CD45-PE-Cy5 (clone 30-F11, Thermo Fisher Scientific), and-CRTh2-PE-Cy7 (clone BM16, BioLegend).

ELISA. Levels of human IL-5, -IL-3, and -GM-CSF in the serum, and levels of human IL-13, -IL-9, $-\mathrm{IL}-33,-\mathrm{IgE}$, and -eotaxin-1 and $-2, \mathrm{EDN}$ and murine periostin and eotaxin-1 and -2 , in the BALF, of the humanized mice were measured using an ELISA kit for human IL-5, -IL-3, -GM-CSF, and -IL-13, and murine periostin (R\&D Systems) for human IL-9 and human and murine eotaxin-1 and -2 (Abcam), for human IgE (Bethyl Laboratories Inc.), and for human EDN (MBL Co., Ltd.) according to the manufacturer's instructions. In some experiments, the serum monomeric periostin was measured by a sandwich ELISA using 2 anti-periostin mAbs (clones 19D and 19C) as described previously (55).

Multiplex bead-based cytokine immunoassay. A BD CBA Human Cytokine or Chemokine Kit (BD Biosciences) was used to quantify human IFN- $\gamma$, IL-4, IL-1 $\beta$, IL-13, IL-5, IL-12, TNF $\alpha$, CCL2, IL- 8 , and RANTES levels in plasma, following the manufacturer's instructions. The data were acquired using a BD FACS Canto flow cytometry system (BD Biosciences) and analyzed using BD FCAP Array v. 3.0 software (BD Biosciences). A standard curve was generated using a 5-parameter logistic (5-PL) equation.

Histology. For histological analysis, the lungs of the humanized mice were fixed in $10 \%$ buffered formalin (Wako) overnight and embedded in paraffin. Sections 3- $\mu \mathrm{m}$ thick were placed on aminosilane-coated glass slides (Muto Pure Chemicals). Immunohistochemical staining for T cells and mast cells was performed using a Leica Bond-Max automated staining system (Leica Biosystems). Paraffin sections were dewaxed in Bond Dewax solution (Leica Biosystems) and dehydrated by incubation in alcohol. After antigen retrieval in 10 $\mathrm{mM}$ citrate buffer ( $\mathrm{pH}$ 6.0), the sections were stained with anti-human CD3 (clone SP7, Nichirei Biosciences, Tokyo, Japan) and mast cell chymase (MCC) antibodies (clone CC1, Leica Biosystems). Detection and 
color development, including hematoxylin counterstaining, were performed using the Bond Polymer Refine Detection system with the Leica Bond-Max automatic immunostainer (Leica Biosystems). Immunohistochemical staining for periostin (rabbit anti-periostin polyclonal antibody; made in house) was performed as described previously (56). To detect mucin-producing goblet cells, the sections were stained with Alcian Blue (AB) (pH 2.5; Muto Pure Chemicals) and PAS (Muto Pure Chemicals) and washed with sulfuric acid. Nuclei were visualized by staining with hematoxylin.

Measurement of AHR. One day after final treatment of human IL-33, mice were i.p. anesthetized with urethane $(1.8 \mathrm{~g} / \mathrm{kg})$ and pancuronium bromide $(0.8 \mathrm{mg} / \mathrm{kg})$ (MilliporeSigma). Airway resistance $(\mathrm{R})$ after delivery of aerosolized methacholine $(0-50 \mathrm{mg} / \mathrm{ml})$ was determined using the FinePointe airway resistance analysis system (DSI Inc.).

Treatment with an anti-IL-13 antibody. HSC-reconstituted humanized mice were i.p. administered $50 \mu \mathrm{g}$ of anti-human IL-13 antibody (clone JES10-5A2, BioLegend) or rat IgG1 isotype control antibody (clone RTK2071, BioLegend) beginning 1 day before IL-33 intratracheal administration every day for 4 days. The mice were euthanized 1 day after the final administration of anti-IL-13 antibody.

Characterization of human eosinophils. PB samples were collected from the orbital vein of hu-NOG IL-5 Tg or hu-NOG IL-3/GM/IL-5 Tg mice under anesthesia. Single-cell suspensions were prepared using $\mathrm{BD}$ Pharm Lyse (BD Biosciences), and $\mathrm{CD} 6 \mathrm{~b}^{+} \mathrm{CD} 16^{-}$eosinophils were enriched using a FACS AriaII cell sorter (BD Biosciences). The purity of $\mathrm{CD}_{6} 6 \mathrm{~b}^{+} \mathrm{CD} 16^{-}$cells was typically $90 \%-95 \%$. For May-Grünwald Giemsa staining, the enriched subpopulations were precipitated onto silane-coated glass slides (Muto Pure Chemicals) by cytospinning and air-dried for 3 minutes. The slides were soaked in May-Grünwald solution (Muto Pure Chemicals) for 3 minutes and washed under running water to remove excess stain. The slides were next stained with $0.5 \%$ Giemsa solution (Muto Pure Chemicals) for 15 minutes. After washing with running water, the slides were dried and subjected to microscopy.

Human and murine eosinophils from BALF were simultaneously quantified by microscopy because they are easily distinguishable by their morphology (57) (Supplemental Figure 1). To stain MBP, cells from PB of hu-IL-5 Tg mice (12 weeks after HSC transplantation) were fixed and permeabilized in PFA (BioLegend) and permeabilization wash buffer (BioLegend). Next, human MBP was stained with a mouse anti-human eosinophil MBP antibody (clone BMK13, MilliporeSigma), followed by antimouse IgG1-APC (clone RMG1-1, BioLegend). Human IgG1-APC (clone QA16A12, BioLegend) was used as an isotype control.

Statistics. Numerical data are presented as means \pm SD unless otherwise noted. Two-tailed Student's $t$ tests or 1-way ANOVA were performed using Excel (Microsoft) or Prism 6 (GraphPad) software. Pearson's correlation coefficient $\left(\mathrm{R}^{2}\right)$ was calculated using Prism6 (GraphPad). Significant differences are indicated as ${ }^{*} P<0.05,{ }^{* *} P<0.005,{ }^{* * *} P<0.0005$, and $* * * * P<0.00005$.

Study approval. All animal experiments were approved by the IACUC of the CIEA (certification number 11004A, February 16, 2011) or by the Animal Experimentation Committee of Nihon University and were performed in accordance with the guidelines of each institute. All experiments using human resources were approved by the Ethical Committee of the CIEA (certification number 08-11, September 4, 2008) and performed in accordance with CIEA guidelines. Written informed consent was obtained from all subjects.

\section{Author contributions}

RI, SM, and SN conceived and designed the study. RI, KS, IK, KK, MY, AH, ST, and SN performed the experiments. RI, IK, AH, TO, MG, and RT developed and maintained the mice. RI, SM, TT, YO, KI, YG, $\mathrm{SH}, \mathrm{MI}$, and SN participated in the analysis and discussions of the results. RI and SN wrote the paper.

\section{Acknowledgments}

We thank Yasuhiko Ando of JAC Inc. and Iyo Otsuka, Yuyo Ka, Kayo Tomiyama, and Takuma Mizusawa of CIEA for maintaining and producing experimental animals; Tsutomu Kamisako and Takahiro Kagawa of CIEA for generating Tg mice; and Miho Miyake and Maki Watanabe of Saga Medical School for technical assistance. This work was supported by Grants-in-Aid for Scientific Research (S) (no. 2222007 to MI) and Grants-in-Aid for Scientific Research (C) (no. 25430099 to RI) from the Japan Society for the Promotion of Science (JSPS) and in part by a Grant-in-Aid for Research on Hepatitis (to TT) from the Japan Agency for Medical Research and Development (AMED) and a research grant (to $\mathrm{SN}$ ) from Novartis Pharma. 
Address correspondence to: Satoshi Nunomura, Division of Medical Biochemistry, Department of Biomolecular Sciences, Saga Medical School, 5-1-1, Nabeshima, Saga, 849-8501, Japan. Phone: 81.952.34.2269; Email: nunomura@cc.saga-u.ac.jp. Or to: Ryoji Ito, Central Institute for Experimental Animals, 3-25-12 Tonomachi, Kawasaki-ku, Kawasaki, Kanagawa 210-0821, Japan. Phone: 81.44.201.8537; Email: rito@ciea.or.jp.

1. Graham LM, Eid N. The impact of asthma exacerbations and preventive strategies. Curr Med Res Opin. 2015;31(4):825-835.

2. O’Byrne PM. Therapeutic strategies to reduce asthma exacerbations. J Allergy Clin Immunol. 2011;128(2):257-263.

3. Frois C, Wu EQ, Ray S, Colice GL. Inhaled corticosteroids or long-acting beta-agonists alone or in fixed-dose combinations in asthma treatment: a systematic review of fluticasone/budesonide and formoterol/salmeterol. Clin Ther. 2009;31(12):2779-2803.

4. Peters SP, Ferguson G, Deniz Y, Reisner C. Uncontrolled asthma: a review of the prevalence, disease burden and options for treatment. Respir Med. 2006;100(7):1139-1151.

5. Holgate ST, Polosa R. The mechanisms, diagnosis, and management of severe asthma in adults. Lancet. 2006;368(9537):780-793.

6. Kabata H, et al. Thymic stromal lymphopoietin induces corticosteroid resistance in natural helper cells during airway inflammation. Nat Commun. 2013;4:2675.

7. Oboki K, Nakae S, Matsumoto K, Saito H. IL-33 and Airway Inflammation. Allergy Asthma Immunol Res. 2011;3(2):81-88

8. Saluja R, Khan M, Church MK, Maurer M. The role of IL-33 and mast cells in allergy and inflammation. Clin Transl Allergy. 2015;5:33.

9. Yao X, Sun Y, Wang W, Sun Y. Interleukin (IL)-25: Pleiotropic roles in asthma. Respirology. 2016;21(4):638-647.

10. Lloyd CM, Hessel EM. Functions of T cells in asthma: more than just T(H)2 cells. Nat Rev Immunol. 2010;10(12):838-848.

11. Bagnasco D, Ferrando M, Varricchi G, Passalacqua G, Canonica GW. A critical evaluation of anti-IL-13 and anti-IL-4 strategies in severe asthma. Int Arch Allergy Immunol. 2016;170(2):122-131.

12. Wenzel S, et al. Dupilumab efficacy and safety in adults with uncontrolled persistent asthma despite use of medium-to-highdose inhaled corticosteroids plus a long-acting $\beta 2$ agonist: a randomised double-blind placebo-controlled pivotal phase $2 \mathrm{~b}$ dose-ranging trial. Lancet. 2016;388(10039):31-44.

13. Wenzel S, et al. Dupilumab in persistent asthma with elevated eosinophil levels. N Engl J Med. 2013;368(26):2455-2466

14. Zosky GR, Sly PD. Animal models of asthma. Clin Exp Allergy. 2007;37(7):973-988.

15. Zosky GR, von Garnier C, Stumbles PA, Holt PG, Sly PD, Turner DJ. The pattern of methacholine responsiveness in mice is dependent on antigen challenge dose. Respir Res. 2004;5:15.

16. Fattouh R, et al. House dust mite facilitates ovalbumin-specific allergic sensitization and airway inflammation. Am J Respir Crit Care Med. 2005;172(3):314-321.

17. Johnson JR, et al. Continuous exposure to house dust mite elicits chronic airway inflammation and structural remodeling. $A m J$ Respir Crit Care Med. 2004;169(3):378-385.

18. Lundy SK, Berlin AA, Lukacs NW. Interleukin-12-independent down-modulation of cockroach antigen-induced asthma in mice by intranasal exposure to bacterial lipopolysaccharide. Am J Pathol. 2003;163(5):1961-1968.

19. Mehlhop PD, et al. Allergen-induced bronchial hyperreactivity and eosinophilic inflammation occur in the absence of IgE in a mouse model of asthma. Proc Natl Acad Sci U S A. 1997;94(4):1344-1349.

20. Fan M, Jamal Mustafa S. Role of adenosine in airway inflammation in an allergic mouse model of asthma. Int Immunopharmacol. 2006;6(1):36-45.

21. Ito M, et al. NOD/SCID/gamma(c)(null) mouse: an excellent recipient mouse model for engraftment of human cells. Blood 2002;100(9):3175-3182.

22. Ito M, Kobayashi K, Nakahata T. NOD/Shi-scid IL2rgamma(null) (NOG) mice more appropriate for humanized mouse models. Curr Top Microbiol Immunol. 2008;324:53-76.

23. Ito R, et al. Efficient xenoengraftment in severe immunodeficient NOD/Shi-scid IL2rynull mice is attributed to a lack of CD11 ${ }^{+}$B220 ${ }^{+}$CD122 ${ }^{+}$cells. J Immunol. 2012;189(9):4313-4320.

24. Shultz LD, et al. Human lymphoid and myeloid cell development in NOD/LtSz-scid IL2R $\gamma$ null mice engrafted with mobilized human hemopoietic stem cells. J Immunol. 2005;174(10):6477-6489.

25. Ishikawa F, Saito Y, Yoshida S, Harada M, Shultz LD. The differentiative and regenerative properties of human hematopoietic stem/progenitor cells in NOD-SCID/IL2ry(null) mice. Curr Top Microbiol Immunol. 2008;324:87-94.

26. Traggiai E, et al. Development of a human adaptive immune system in cord blood cell-transplanted mice. Science. 2004;304(5667):104-107.

27. Ito R, et al. Establishment of a human allergy model using human IL-3/GM-CSF-transgenic NOG mice. J Immunol. 2013;191(6):2890-2899.

28. Ito R, et al. A novel in vivo model for predicting myelotoxicity of chemotherapeutic agents using IL-3/GM-CSF transgenic humanized mice. Toxicol Lett. 2017;281:152-157.

29. Takayama G, et al. Periostin: a novel component of subepithelial fibrosis of bronchial asthma downstream of IL-4 and IL-13 signals. J Allergy Clin Immunol. 2006;118(1):98-104.

30. Izuhara K, et al. Periostin in inflammation and allergy. Cell Mol Life Sci. 2017;74(23):4293-4303.

31. Woodruff PG, et al. T-helper type 2-driven inflammation defines major subphenotypes of asthma. Am J Respir Crit Care Med. 2009;180(5):388-395.

32. Gour N, Wills-Karp M. IL-4 and IL-13 signaling in allergic airway disease. Cytokine. 2015;75(1):68-78.

33. Varricchi G, Senna G, Loffredo S, Bagnasco D, Ferrando M, Canonica GW. Reslizumab and eosinophilic asthma: one step closer to precision medicine? Front Immunol. 2017;8:242.

34. Zhang JY, Wenzel SE. Tissue and BAL based biomarkers in asthma. Immunol Allergy Clin North Am. 2007;27(4):623-632.

35. Acharya KR, Ackerman SJ. Eosinophil granule proteins: form and function. J Biol Chem. 2014;289(25):17406-17415.

36. Renauld JC. New insights into the role of cytokines in asthma. J Clin Pathol. 2001;54(8):577-589. 
37. Snelgrove RJ, et al. Alternaria-derived serine protease activity drives IL-33-mediated asthma exacerbations. J Allergy Clin Immunol. 2014;134(3):583-592.e6.

38. Mizutani N, Nabe T, Yoshino S. IL-17A promotes the exacerbation of IL-33-induced airway hyperresponsiveness by enhancing neutrophilic inflammation via CXCR2 signaling in mice. J Immunol. 2014;192(4):1372-1384.

39. Nagarkar DR, et al. IL-13 mediates IL-33-dependent mast cell and type 2 innate lymphoid cell effects on bronchial epithelial cells. J Allergy Clin Immunol. 2015;136(1):202-205.

40. Christianson CA, et al. Persistence of asthma requires multiple feedback circuits involving type 2 innate lymphoid cells and IL-33. J Allergy Clin Immunol. 2015;136(1):59-68.e14.

41. Rigas $\mathrm{D}$, et al. Type 2 innate lymphoid cell suppression by regulatory $\mathrm{T}$ cells attenuates airway hyperreactivity and requires inducible T-cell costimulator-inducible T-cell costimulator ligand interaction. J Allergy Clin Immunol. 2017;139(5):1468-1477.e2.

42. Lim AI, et al. Systemic human ILC precursors provide a substrate for tissue ILC differentiation. Cell. 2017;168(6):1086-1100.e10

43. Sidhu SS, et al. Roles of epithelial cell-derived periostin in TGF-beta activation, collagen production, and collagen gel elasticity in asthma. Proc Natl Acad Sci U S A. 2010;107(32):14170-14175.

44. Corren J, et al. Lebrikizumab treatment in adults with asthma. N Engl J Med. 2011;365(12):1088-1098.

45. Cho JY, et al. Inhibition of airway remodeling in IL-5-deficient mice. J Clin Invest. 2004;113(4):551-560

46. Flood-Page P, et al. Anti-IL-5 treatment reduces deposition of ECM proteins in the bronchial subepithelial basement membrane of mild atopic asthmatics. J Clin Invest. 2003;112(7):1029-1036.

47. Legrand F, Klion $\mathrm{AD}$. Biologic therapies targeting eosinophils: current status and future prospects. J Allergy Clin Immunol Pract 2015;3(2):167-174.

48. Li L, et al. Effects of Th2 cytokines on chemokine expression in the lung: IL-13 potently induces eotaxin expression by airway epithelial cells. J Immunol. 1999;162(5):2477-2487.

49. Yoshida T, et al. Defective B-1 cell development and impaired immunity against Angiostrongylus cantonensis in IL-5R alpha-deficient mice. Immunity. 1996;4(5):483-494.

50. Hammad H, Chieppa M, Perros F, Willart MA, Germain RN, Lambrecht BN. House dust mite allergen induces asthma via Toll-like receptor 4 triggering of airway structural cells. Nat Med. 2009;15(4):410-416.

51. Watanabe Y, et al. The analysis of the functions of human B and T cells in humanized NOD/shi-scid/gammac(null) (NOG) mice (hu-HSC NOG mice). Int Immunol. 2009;21(7):843-858.

52. Suzuki M, et al. Induction of human humoral immune responses in a novel HLA-DR-expressing transgenic NOD/Shi-scid/ $\gamma$ cnull mouse. Int Immunol. 2012;24(4):243-252.

53. Ito R, Takahashi T, Katano I, Ito M. Current advances in humanized mouse models. Cell Mol Immunol. 2012;9(3):208-214.

54. Maruoka S, et al. Involvement of apoptosis signal-regulating kinase-1 in house dust mite-induced allergic asthma in mice. Allergol Int. 2017;66S:S50-S52.

55. Sugiyama A, et al. Periostin promotes hepatic fibrosis in mice by modulating hepatic stellate cell activation via $\alpha \mathrm{v}$ integrin interaction. J Gastroenterol. 2016;51(12):1161-1174.

56. Ontsuka K, et al. Periostin, a matricellular protein, accelerates cutaneous wound repair by activating dermal fibroblasts. Exp Dermatol. 2012;21(5):331-336.

57. Lee JJ, et al. Human versus mouse eosinophils: "that which we call an eosinophil, by any other name would stain as red". $J$ Allergy Clin Immunol. 2012;130(3):572-584. 